OPEN ACCESS

Edited by:

Tao Jiang,

Capital Medical University, China

Reviewed by:

Thomas Wieland,

Heidelberg University, Germany

Maria Giuseppina Miano,

Institute of Genetics and Biophysics

(CNR), Italy

*Correspondence:

Veronica Ferrucci

veronica.ferrucci@unina.it

Massimo Zollo

massimo.zollo@unina.it

${ }^{t}$ These authors have contributed equally to this work

Specialty section:

This article was submitted to

Neuro-Oncology and

Neurosurgical Oncology,

a section of the journal

Frontiers in Oncology

Received: 13 August 2021 Accepted: 28 September 2021

Published: 22 October 2021

Citation:

Bibbò F, Sorice C, Ferrucci V and Zollo M (2021) Functional

Genomics of PRUNE1 in

Neurodevelopmental Disorders (NDDs) Tied to Medulloblastoma

(MB) and Other Tumors.

Front. Oncol. 11:758146. doi: 10.3389/fonc.2021.758146

\section{Functional Genomics of PRUNE1 in Neurodevelopmental Disorders (NDDs) Tied to Medulloblastoma (MB) and Other Tumors}

\author{
Francesca Bibbò $^{1,2+}$, Carmen Sorice ${ }^{1,2 \dagger}$, Veronica Ferrucci ${ }^{1,2 *}$ and Massimo Zollo ${ }^{1,2 *}$ \\ ${ }^{1}$ Dipartimento di Medicina Molecolare e Biotecnologie Mediche (DMMBM), 'Federico II' University of Naples, Naples, Italy, \\ ${ }^{2}$ CEINGE Biotecnologie Avanzate, Naples, Italy
}

We analyze the fundamental functions of Prune_1 in brain pathophysiology. We discuss the importance and maintenance of the function of Prune_1 and how its perturbation influences both brain pathological conditions, neurodevelopmental disorder with microcephaly, hypotonia, and variable brain anomalies (NMIHBA; OMIM: 617481), and tumorigenesis of medulloblastoma (MB) with functional correlations to other tumors. A therapeutic view underlying recent discoveries identified small molecules and cell penetrating peptides to impair the interaction of Prune_1 with protein partners (e.g., $\mathrm{Nm} 23-\mathrm{H} 1$ ), thus further impairing intracellular and extracellular signaling (i.e., canonical Wnt and TGF- $\beta$ pathways). Identifying the mechanism of action of Prune_1 as responsible for neurodevelopmental disorders (NDDs), we have recognized other genes which are found overexpressed in brain tumors (e.g., MB) with functional implications in neurodevelopmental processes, as mainly linked to changes in mitotic cell cycle processes. Thus, with Prune_1 being a significant target in NDDs, we discuss how its network of action can be dysregulated during brain development, thus generating cancer and metastatic dissemination.

\begin{abstract}
Keywords: PRUNE_1, medulloblastoma, metastasis, proliferation rate, microtubules polymerization, Wnt, TGFtransforming growth factor, neurodevelopmental disorder with microcephaly, hypotonia, variable brain anomalies (NMIHBA)
\end{abstract}

\section{INTRODUCTION}

PRUNE_1, the human homolog of the Drosophila melanogaster pn gene, was firstly identified by viable mutations responsible for the brunish purple eye color compared to the red of wild-type fly (1). The phenotype of $p n$ mutant flies was due to alterations in the metabolism of drosopteridines (1). Interestingly, the same homozygous or hemizygous $p n$ mutation became lethal when the protein encoded by abnormal wing disc (i.e., awd) gene was also mutated (i.e., p.P97S) (2). Awd is the homolog of the human NME1 gene encoding for the Nm23-H1 (or NDPK-A) protein, one of the known Prune_1 binders. In this regard, the double mutant flies died at the second/third larval developmental stage because of anomalies of mesoderm development and central nervous system (CNS)-like cells. Interestingly, the mutant p.P97S awd protein still retained the ability to bind 
Prune_1 protein (3). Thus, the "lethal interaction" between awd and pn mutant proteins was hypothesized to be due to neomorphic mutations unbalancing the switch from guanosine triphosphate (GTP) to guanosine diphosphate (GDP; mainly mediated by awd protein), thus causing alterations in the modulation of GTPases and/or GTPase-activating proteins (4).

Importantly, Prune-M1 and Nm23-M1 (the mouse homologs of the human PRUNE_1 and NME1 genes, respectively) showed significant co-expression patterns in the cortex, hippocampus, midbrain, and the cerebellum during murine neural development, thus suggesting a critical role for these proteins during neural development (5).

The human Prune_1 protein belongs to the DHH (AspHis-His) protein superfamily (that also includes the $p n$ protein of Drosophila and the RecJ exonuclease of bacteria) and possesses a nucleotide phosphodiesterase (PDE) (6) and an exopolyphosphatase (exopolyphosphatase/pyrophosphatase, PPX/PPase) action, with greater affinity for short-chain over long-chain inorganic polyphosphates (polyPs) (7). Regarding its tertiary structure, human Prune_1 is a naturally unfolded protein with the ability to interact with several intracellular binding partners, including GSK-3 $\beta$ (8) and Nm23-H1/H2 (9). Furthermore, Prune_1 was also identified as a microtubuleassociated protein (MAP) with a role in the enhancement of microtubule (MT) polymerization in the mitotic spindle during cell division (10).

Because of the enzymatic activities and its interaction with several proteins, Prune_1 was found able to modulate both intraand extracellular signaling cascades [including the canonical Wnt (8) and TGF- $\beta$ (11) pathways] that regulate cell proliferation, motility, and epithelial-mesenchymal transition (EMT) processes.

To date, Prune_1 has been found highly expressed and positively correlated with the grading, EMT, and metastatic status in several tumors, including those of the CNS and the peripheral nervous system (PNS), and in medulloblastoma (MB) and neuroblastoma (NBL). Indeed, high expression levels of Prune_1 were found in metastatic MB (11), the most common childhood brain tumor. In MB group 3, Prune_1 drives a "metastatic axis," thus leading to the enhancement of the TGF$\beta$ pathway, activation of EMT process, and the reduction of the amount of PTEN protein (11).

Furthermore, recessive mutations in PRUNE_1 locus (1q21.3) were also identified as candidate genetic causes of neurodevelopmental disorder with microcephaly, hypotonia, and variable brain anomalies (NMIHBA; MIM \#617481) (10, 12-20). Similarly, other genes responsible for microcephaly $(\mathrm{MCPH})$ and mainly implicated in cell division mechanisms were also found both overexpressed in brain tumors (i.e., MB) and mutated in neurodevelopmental disorders (NDDs).

In this review, we will focus on the Prune_1 protein enzymatic activities, intracellular pathways, and protein interactors in the interplay between metastatic CSN tumor (i.e., $\mathrm{MB}$ ) and NDD (i.e., NMIHBA) pathogenesis. In conclusion, we also highlight the use of Prune_1-inhibitors as new potential therapies for the treatment of brain tumors. In the near future, once the mechanisms have been identified, we will then use this knowledge to understand their use and potential application in therapeutic interventions also in NDDs.

\section{PRUNE 1 ENZYMATIC ACTIVITIES}

Prune_1 protein is part of DHH phosphoesterase superfamily. Its $\mathrm{N}$-terminal domain contains the DHH motifs (6). Prune_1 retains two different enzymatic functions-PDE (6) and PPX/ PPase activities - the latest with an order of magnitude higher (7).

\section{Phosphodiesterase Activity}

Regarding PDE activity, Prune_1 has the ability to hydrolyze the second messengers adenosine and guanosine $3^{\prime}, 5^{\prime}$-cyclic nucleotides [i.e., cyclic adenosine monophosphate (cAMP) and cyclic guanosine monophosphate (cGMP)] in their corresponding 5'-monophosphates (i.e., 5'-AMP and 5'-GMP, respectively). cAMP has major affinity to Prune_1, then cGMP ( $K_{\mathrm{m}}$ values: cAMP, $0.9 \pm 0.03 \mathrm{M}$; cGMP, $\left.2.3 \pm 0.11 \mathrm{M}\right)$ (6). Phosphodiesterase enzymes have important roles in cellular homeostasis because of the pivotal functions of cAMP and cGMP (as second messengers) in physiological processes due to the modulation of signaling pathway transduction (21). Furthermore, Prune_1 PDE activity has been reported to enhance cell motility in vitro (6). In this regard, MBA-MB-435 cells that overexpressed mutant Prune_1 proteins lacking PDE activity [through amino acid changes within the region containing motif III (DHRP126-129AAAA, Prunes) were found with decreased migration rates (6). Furthermore, dipyridamole, a PDE inhibitor, was also found to affect the motility of breast cancer (BC) cells (i.e., MDA-MB231T) (22, 23). These data, thus, suggest that the PDE enzymatic activity of Prune_1 protein may have a role in the modulation of the migratory properties of tumorigenic cells in which Prune_1 is overexpressed.

\section{Exopolyphosphatase (PPX/PPase) Activity}

The PPX/PPase (polyphosphate-phosphohydrolases) activity of Prune_1 was found to exceed its PDE function (7). Prune_1 has affinity for short-chain over long-chain inorganic polyPs. Indeed, it can hydrolyze linear polyPs by using $\mathrm{Mg}^{2+}$ or $\mathrm{Co}^{2+}$ as a cofactor. Among the inorganic polyPs with different chain lengths, the highest $K_{\text {cat }}$ values for Prune_1 were observed by using triphosphates (P3), adenosine $5^{\prime}$-tetraphosphate (AP4), and guanosine $5^{\prime}$-tetraphosphate (GP4) as substrates. These values dramatically decreased with polyPs at increased chain lengths. Interestingly, PPi, ATP, and GTP were not significantly hydrolyzed (7). Of importance is that loss of $\mathrm{PPX} / \mathrm{PPase}$ activity was found in the recombinant mutants p.N24H, p.D28A, p.D179A, and p.R348A Prune_1 proteins when assayed in the presence of $\mathrm{P} 3$ and $\mathrm{Mg}^{2+}$ as the substrate and cofactor, respectively. The other mutants, p.D106A, p.H107N, and p.H108N Prune_1 proteins, had reduced but measurable $\mathrm{PPX} / \mathrm{PPase}$ activity. In contrast, the mutant 
p.R128H Prune_1 protein showed an increased $K_{\text {cat }}$ value in comparison with the wild-type protein (7). These results suggest that variations within the $\mathrm{DHH}$ domain (e.g., p.D106A, p.H107N, and p.H108N) did not affect the PPX/ PPase activity of Prune_1 (7). In contrast, the same PPX/ PPase enzymatic function resulted affected in mutant Prune_1 proteins containing variations in those residues conserved in PPase and PPX enzymes (i.e., p.D28A, p.D179A, and p.R348A) (7).

At this time, questions were raised about the potential role of PPX/PPase activity in cell motility. In this regard, a delayed migratory rate was shown in $\mathrm{HaCaT}$ cells overexpressing PPX1 (the Saccharomyces cerevisiae homolog of the human PRUNE_1 gene) by performing would healing assays (24). However, different functions were also postulated due to the role that long-chain polyPs play in the induction of ERK1-2 (i.e., MAP kinase pathway) in human cells (25). Thus, how the PPX/PPAse activity of Prune_1 influences migratory processes and cell motility in both physiological conditions is still not fully understood (in the context of polyP degradation and/or organelle cellular compartment storage). Future research studies and discoveries will address these questions.

Inorganic polyPs are polymers of linear orthophosphate (Pi) units ranging from five and several thousand orthophosphates that are linked through phosphoanhydride bonds (one of the most energy-rich linkages). In human, their amounts are very high in blood plasma, platelets, and osteoblasts, where they modulate blood clotting (26), mineralization processes, and the regulation of ATP level (27). Thus, polyPs are involved in many functions mostly related to additional sources of energy into the cells. Interestingly, polyPs were found to act as cytoprotective agents against human immunodeficiency virus type 1 (HIV-1) (28). In this regard, very recently, polyPs have been found able to exert antiviral actions against severe acute respiratory syndrome coronavirus 2 (SARS-CoV-2) (29), during early phase of infection and replication (30).

Furthermore, high concentrations of polyPs were also found in mammalian brain, especially in astrocytes, where they were found to induce calcium signaling through P2Y1 purinergic receptors and phospholipase $\mathrm{C}$ induction, inositol 3 phosphate (IP3) delivery, and cytosolic $\mathrm{Ca}^{2+}$ increase (31, 32). Moreover, PolyPs were also reported to amplify the proinflammatory response in endothelial cells via the activation of the same P2Y1 receptor (33), thus overall modulating mitochondrial functions. In this regard, the depletion of mitochondrial polyPs (through the overexpression and the activity of yeast PPX) significantly increased the levels of orthophosphates in the mitochondrial matrix (which is necessary for $\mathrm{Ca}^{2+}$ binding), thus resulting in a decreased mitochondrial membrane potential and the inhibition of complex I of the respiratory chain $(34,35)$. Thus, the crucial role of polyPs in cell metabolism is clear, as also confirmed in transgenic mice widely expressing the scPPX1 exopolyphosphatase that displayed a reduced mitochondrial respiration in muscles (36).

\section{PRUNE_1 PROTEIN INTERACTION WITH PROTEIN BINDING PARTNERS}

Human Prune_1 is a naturally unfolded multi-domain adaptor protein. The three-dimensional structure of the carboxylterminus (C-terminus) region of Prune_1 was obtained through nuclear magnetic resonance (NMR) studies and molecular dynamics techniques (37). Two globular regions responsible for its enzymatic activities (i.e., DHH and DHHA2 domains) were found within the amino-terminus (N-terminus) domain of Prune_1 (37). The DHHA2 domain also contained a conserved DXK motif at its N-terminus. An intrinsically disordered domain (starting at 371 amino acid residue) containing two stretches with a tendency for helix structures was also identified. The C-terminal domain of Prune_1 is characterized by residues (postulated to reside between 393 and 420) that are involved in its homodimerization and a small globular region responsible for its interactions with other proteins $(38,39)$. In fact, Prune_1 was found with the ability to bind several intracellular protein partners mainly involved in cytoskeletal rearrangement, including $\alpha$ - and $\beta$-tubulins, glycogen synthase kinase $3 \beta$ (GSK-3 $\beta$ ) (40), and Nm23-H1/H2 (or NDPK-A/B) (6). Through these interactions, Prune_1 can modulate different intracellular pathways that might be then also translated into extracellular signaling, including the canonical Wnt via GSK-3 $\beta$ (8) and TGF- $\beta$ via Nm23-H1 binding (11).

\section{Prune_1 Interaction With GSK- $\beta$}

The region of Prune_1 responsible for the interaction with GSK$3 \beta$ starts from 330 to 394 amino acid residues within the Cterminus domain $(8,40)$. Because of the co-localization between Prune_1 and the focal adhesion proteins (i.e., F-actin, paxillin, and vinculin) in several cell types, this Prune_1-GSK-3 $\beta$ interaction suggests that Prune_1 can also modulate the microtubular architecture and dynamics (40-42). Importantly, GSK-3 $\beta$ is considered a negative modulator of the canonical WNT/ $\beta$-catenin pathway through phosphorylation at its amino acid residues S9 and S21 $(8,9)$. Of importance is that, through GSK-3 $\beta$ interaction, Prune_1 was found to activate $\beta$-catenin signaling cascade and to further promote Wnt3a secretion in non-small cell lung cancer (NSCLC) cells (8); thus, a connectome related to the WNT signaling activation was identified (43).

\section{Prune_1 Interaction With NME-1 (Nm23-H1)}

Prune_1 was also shown to physically interact with the Cterminus domain of Nm23-H1 through its D388 and D422 residues (37). Nm23-H1 is described as the first anti-metastatic gene with different functions: a nucleoside diphosphate kinase (NDPK) activity that catalyzes the transfer of a phosphoryl group from a nucleoside triphosphate (NTP) to a nucleotide diphosphate (NDP), a histidine kinase function, and a $3^{\prime}-5^{\prime}$ exonuclease activity (44). In detail, in vitro studies demonstrated that the complex formation involved the dimer of Prune_1 and the hexameric form of Nm23-H1 (38). Interestingly, Nm23-H1 and GSK-3 $\beta$ could bind Prune_1 at the same time by using non- 
identical regions of its C-terminus domain for their binding, thus suggesting two independent interaction sites for signaling complex assembly (38). Moreover, other in vitro studies demonstrated that the Prune_1/Nm23-H1 interaction complex requires casein kinase I/II-mediated phosphorylation on three serine residues (S120, S122, and S125) of Nm23-H1. Phosphorylated Nm23-H1 on S120, S122, and S125 was also found to increase its hexameric form (41). Of importance the overexpression of both Prune_1 and Nm23-H1 was found in different tumors, such as MB (11), NBL (37) and BC (45). In this regard, the data indicate that the Prune_1/Nm23-H1 complex could affect the anti-metastatic activity of $\mathrm{Nm} 23-\mathrm{H} 1$ and increase the cell motility properties $(45,46)$.

\section{Prune_1 Interaction With $\alpha$ - and $\beta$-Tubulins}

Other potential Prune_1 interactors were identified through a pull-down assay coupled to mass spectrometry analysis in BC cells (42). Through this approach, new potential Prune_1 binders were identified. Among these, the interaction between Prune_1 and $\beta$-tubulin was proposed because of the crucial functions played by tubulins, MTs, and MAPs in neural development (47, 48). Furthermore, this interaction was also confirmed by coimmunoprecipitation assays (Prune_1 protein and both $\beta$ - and $\alpha$-tubulins) in NBL inducible cell clones (SH-SY5Y) (10). Of significance is that Prune_1 protein was also identified as a MAP by performing a cell-based microtubule-binding protein spindown assay using the same neuronal SH-SY5Y cell clones (10). These data were also confirmed by MT polymerization assays in vitro and "in cells," thus showing an enhancement of the MT polymerization rate (nucleation, growth, and steady-state phases) in the presence of a recombinant wild-type Prune_1 protein (10). Furthermore, Prune_1 was also found to co-localize with MTs (i.e., $\beta$-tubulin) in mitotic spindles during cell division (prometaphase, metaphase, anaphase, and cytokinesis) in Hela cells (10). Taken altogether, these data indicate an important role for Prune_1 protein in cellular division processes.

\section{PRUNE_1 IN NEURODEVELOPMENTAL DISORDER}

\section{The Role of Prune_1 During Neural Development}

Prune_1 was found to take part in fundamental processes occurring during neural development. In this regard, PruneM1 and Nm23-M1 (i.e., the murine homologs of human PRUNE_1 and NME-1, respectively) had similar expression patterns in the developing murine nervous system, from early brain development to adulthood (5). In fact, a significant spatiotemporal co-expression of both Prune-M1 and Nm23-M1 during brain development, within the cortex, hippocampus, and midbrain, was observed. Regarding the telencephalon, both genes were found expressed in the ventricular zone (VZ) of the neopallial cortex, a region involved in proliferation processes. Furthermore, the expressions of Prune-M1 and Nm23-M1 were also shown within the intermediate zone and in the ganglionic eminence. These regions allow neurons to migrate to the pial surface of the forebrain to then undergo differentiation processes. Furthermore, Prune-M1 and Nm23-M1 were also expressed in the cerebellum during development in both granular and Purkinje neuron precursors (5). These findings suggest fundamental functions for Prune_1 and Nm23-H1 during cell proliferation, migration, and differentiation processes occurring in the developing brain and mainly in the cerebellum. Of interest is that the expressions of Prune-M1 and Nm23-M1 were also shown during the murine postnatal phases in cortical layers II and IV and in the basal ganglia of the striatum, thus indicating a potential involvement of the complex in somatosensory processing and in synaptic function and plasticity (5). Interestingly, Nme-X1 and Prune-X1 (i.e., Xenopus homolog proteins) were also reported with a potential function in Muller gliogenesis during retinal development in Xenopus (49). This, thus, indicates a role for the Prune_1 and Nm23-H1 complex also during the development of the retina and in eye morphogenesis.

\section{Recessive Mutations in PRUNE_1 Locus Are Responsible for NDD}

Recently, homozygous and composite heterozygous mutations in PRUNE_1 locus (1q21.3) were found [through whole-exome sequencing (WES) analyses] in several patients worldwide as causative of the autosomal-recessive NMIHBA (MIM \#617481). NMIHBA is a severe disease due to the global developmental delay and the intellectual disability. In fact, most of the affected patients presented $\mathrm{MCPH}$ and brain, cerebellar, and optic atrophy. Other cerebral abnormalities were also reported in these patients (through brain imaging), such as cortical atrophy, thickness of the corpus callosum, and hypoplasia of the cerebellum. Some patients were also described with seizure, peripheral spasticity, and slight delay in myelination process. Furthermore, the phenotype is often accompanied by a decreased muscle tone (i.e., "truncal hypotonia"), impossibility to ambulate, or speech and broad dysmorphism.

This complex phenotypic spectrum in NMIHBA patients can be reasoned by the genotypic diversity in PRUNE_1 locus. To date, 64 patients carrying different mutations in the PRUNE_1 gene have been reported worldwide (Figure $\mathbf{1}$ and Table 1). Regarding the genotypic differences, among the variants identified in patients with PRUNE_1, the most representative was the homozygous c.G316A (p.D106N) variant that was found in 15 subjects: seven from Turkey $(12,16,18)$, three from Italy $(10,14)$, one from Sri Lanka (19), one from Caucasus (17), and three from Lebanon (16). The majority of homozygous mutations were found within the $\mathrm{DHH}$ domain of PRUNE_1, including c.G88A (p.D30N) in six patients from Oman (10) and one from Saudi Arabia (12), c.160C >A (p.P54T) in seven Iranian subjects (10), c.383G $>$ A (p.R128Q) in two patients from Saudi Arabia (15), and c.515T $>C$ (p.L172P) in three children from North Africa (16). Two types of homozygous mutations were also found within the DHHA2 domain: the missense variant c.C889T (p.R297W) in two patients from India (10) and the 

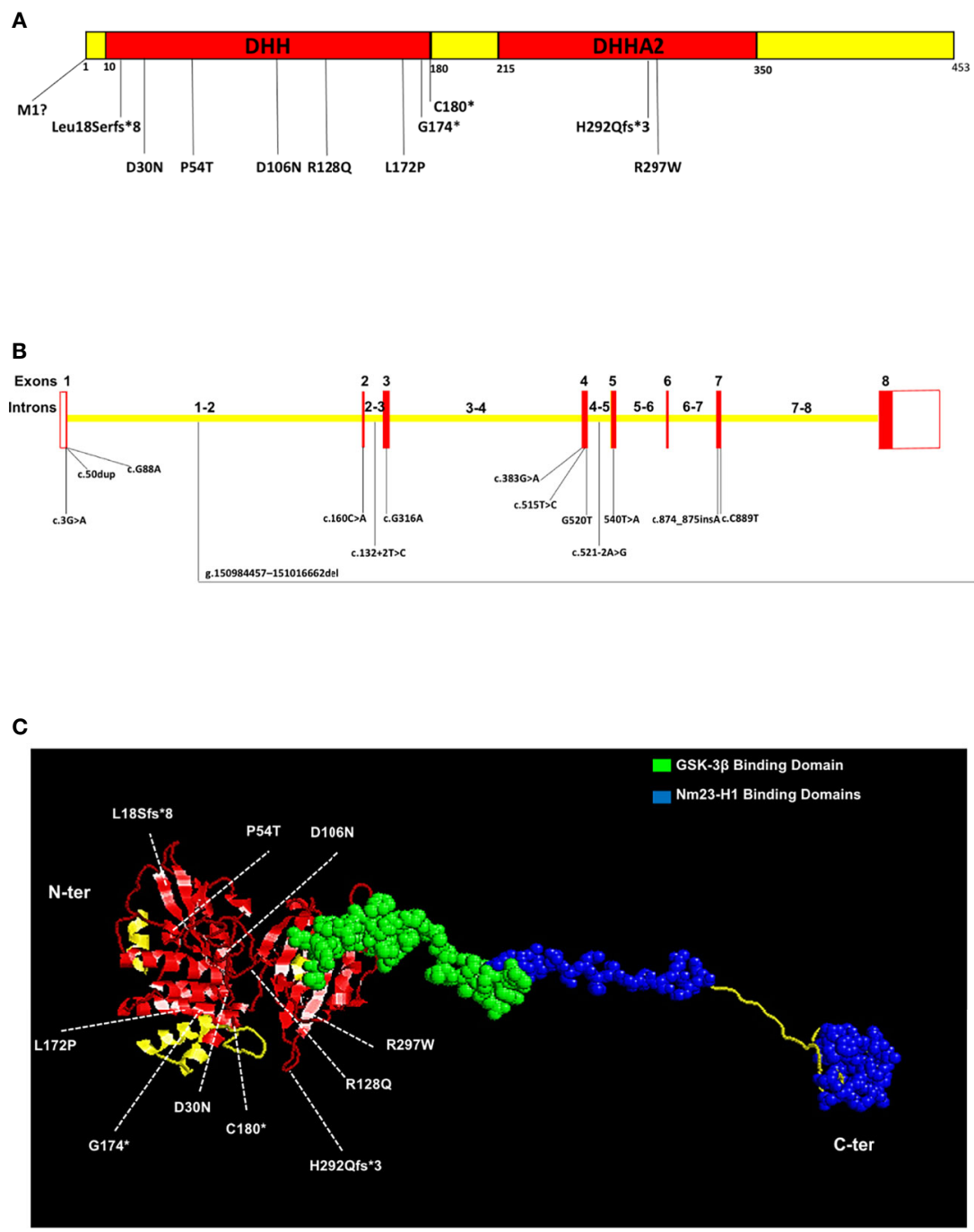

FIGURE 1 | A schematic representation of the Prune_1 protein and PRUNE_1 gene with the mutations and binding regions, as reported in the literature.

(A) Prune_1 protein is composed of 453 amino acids harboring the DHH (Asp-His-His) (from amino acid residues 10 to 180) and DHHA2 (from residues 215 to 350 ) domains. In the $\mathrm{DHH}$ domain, the truncating variant p.L18Sfs 8 , the missense mutations p.M1?, p.D30N, p.P54T, p.D106N, p.R128Q, p.L172P, and the composite heterozygous mutations p.R128Q;G174X, and p.D106N;C180* have been identified. In the DHHA2 domain, the missense variant p.R297W and the homozygous frameshift variant p.H292Qfs*3 have been identified. (B) Exons are denoted by red boxes, UTRs are denoted by white boxes, and introns are denoted by yellow boxes. In exon 1 are shown the homozygous truncating c.50dup variant and the homozygous mutation c.G88A. The homozygous deletion g.1509-84457-151016662del involves exons 2-8. Other homozygous mutations are found in exon 1 (c.3G>A) exon 2 (c.160C>A), exon 3 (c.G316A), and exon 4 (c.383G>A, c.515T>C, and G520T). The mutation C.132+2T>C was found in the splice donor site within intron 2, while the c.521-2A>G variant was found in the canonical splice acceptor site in intron 4. The homozygous frameshift variant c.874_875insA and the missense mutation c.C889T were found in exon 7. (C) Three-dimensional representation of Prune 1 protein from the $\mathrm{N}$-terminal (left) to the C-terminal (right). The DHH (from amino acid residues 10 to 180) and DHH2 (from residues 215 to 350 ) domains are shown in red. The GSK-3 3 binding domain (from residues 330 to 394) is represented in green. The Nm23-H1 binding domains (from amino acid residues 388 to 402 and from 422 to 446) are depicted in blue. We additionally included in both domains of the protein information about the mutations published in the literature. In the DHH domain the truncating variant p.L18Sfs ${ }^{\star}$, the missense mutations p.D30N, p.P54T, p.D106N, p.R128Q, and p.L172P, and the composite heterozygous mutations p.R128Q;G174X and p.D106N;C180* have been represented. In the DHHA2 domain, the missense variant p.R297W and the homozygous frameshift variant p.H292Qfs*3 have been represented.

frameshift variant c.874_875insA (p.H292Qfs*3) in one subject from Turkey (52). Interestingly, different compound heterozygous mutations were also identified, comprising the c.[G383A];[G520T] [p.(R128Q); (G174X)] variant in two affected siblings from the USA (12) and two European patients
(54) and c. [316G $>A] ;[540 \mathrm{~T}>\mathrm{A}]\left[\mathrm{p} .(\mathrm{D} 106 \mathrm{~N}) ;\left(\mathrm{C} 180^{*}\right)\right]$ in two Japanese subjects $(17,20)$. Furthermore, two different homozygous splicing variants were also described in several patients. In detail, a mutation in the splicing acceptor site (canonical) within intron 4 of PRUNE_1 (c.521-2A>G [IVS4- 
TABLE 1 | List of genotyping features of patients affected by neurodevelopmental disorders (NDDs) due to mutations in PRUNE_1 locus.

\begin{tabular}{|c|c|c|c|c|c|c|}
\hline Nucleotide variations & Amino acid variations & Zygosity & Percentage of patients & No. of patients & Ethnicity & References \\
\hline c.50dup & p.Leu18Serfs*8 & Homozygous & 1.56 & 1 & Japan & (17) \\
\hline \multirow[t]{2}{*}{ c.G88A } & p.D30N & Homozygous & 10.94 & 6 & Oman & (10) \\
\hline & & & & 1 & Saudi Arabia & (12) \\
\hline c. $132+2 \mathrm{~T}>\mathrm{C}$ & - & Homozygous & 7.81 & 5 & Sudan & $(50)$ \\
\hline c. $160 \mathrm{C}>\mathrm{A}$ & p.P54T & Homozygous & 10.94 & 7 & Iran & (10) \\
\hline \multirow[t]{5}{*}{ c.G316A } & p.D106N & Homozygous & 23.44 & 7 & Turkey & $(12,16)$ \\
\hline & & & & 3 & Italy & (10) \\
\hline & & & & 1 & Sri Lanka & (19) \\
\hline & & & & 1 & Caucasus & $(17)$ \\
\hline & & & & 3 & Lebanon & $(16)$ \\
\hline c. $383 G>A$ & p.R128Q & Homozygous & 3.13 & 2 & Saudi Arabia & $(15)$ \\
\hline c.515T>C & p.L172P & Homozygous & 4.69 & 3 & North Africa & $(16)$ \\
\hline \multirow[t]{2}{*}{ c. $521-2 A>G$} & - & Homozygous & 15.63 & 1 & Oji-Cree & (13) \\
\hline & & & & 9 & Manitoba-Cree & (51) \\
\hline $540 \mathrm{~T}>\mathrm{A}$ & C180* & Homozygous & 1.56 & 1 & Japan & $(17)$ \\
\hline c.874_875insA & p.H292Qfs*3 & Homozygous & 1.56 & 1 & Turkey & $(52)$ \\
\hline c.C889T & p.R297W & Homozygous & 3.13 & 2 & India & (10) \\
\hline c. $3 G>A$ & M1? & Homozygous & 3.13 & 2 & Iran & (53) \\
\hline g.1509-84457-151016-662del & - & Homozygous & 3.13 & 2 & Austria & (16) \\
\hline c. $[316 \mathrm{G}>\mathrm{A}] ;[540 \mathrm{~T}>\mathrm{A}]$ & [p.(D106N);(C180*)] & Composite heterozygous & 3.13 & 2 & Japan & $(17)$ \\
\hline \multirow[t]{2}{*}{ c.[G383A];[G520T] } & [p.(R128Q);(G174X)] & Composite heterozygous & 6.25 & 2 & Europe & (54) \\
\hline & & & & 2 & USA & $(12)$ \\
\hline
\end{tabular}

$2 \mathrm{~A}>\mathrm{G}]$ ) was found in an Oji-Cree male (13) and in nine patients belonging to four Manitoba-Cree families (51). A different mutation in the splice donor site within intron 2 of the $P R U N E \_1$ gene (i.e., c. $132+2 \mathrm{~T}>\mathrm{C}$ ) was also described in five patients from two consanguineous Sudanese families (50). Moreover, homozygous deletion (g.1509-84457-151016662del) starting from exon 2 to exon 8 including the $3^{\prime} \mathrm{UTR}$ was also found in two female children from Austria (16). Similarly, a homozygous truncating c.50dup (p.Leu18Serfs ${ }^{\star} 8$ ) variant was found in a Japanese child (17). Recently, a start loss c.3G $>\mathrm{A}$ (p.Met1)? variant in the PRUNE_1 gene was also reported in two Iranian patients (53).

A genotype/phenotype correlation study was performed to dissect the function of mutant Prune_1 proteins focusing on the cG88A (p.D30N) and c.C889T (p.R297W) variants (10). Of interest is that the recombinant p.D30N and p.R297W mutant proteins (as synthetized and purified in Escherichia coli) were shown to have an increased PPX/PPase activity in vitro on tetraphosphates (P4) as substrates in comparison to the wild-type protein $\left(K_{\text {cat }} / K_{\mathrm{m}}\right.$ values: wild-type, $0.014 \mathrm{mM} / \mathrm{s}$; p.D30N, $0.312 \mathrm{mM} / \mathrm{s}$; p.R297W, $0.064 \mathrm{mM} / \mathrm{s}$ ), thus suggesting a "gain-of-function" activity. The same mutant Prune_1 proteins (i.e., p.D30N and p.R297W) were also shown to affect both the cell proliferation and migration rates in NBL inducible cell clones (SH-SY5Y) (10). Furthermore, the MT polymerization kinetics was found delayed in the presence of the same mutant Prune_1 proteins both in vitro and in cells (10). Altogether, these in vitro results suggest that the cG88A (p.D30N) and c.C889T (p.R297W) variants in the PRUNE_1 gene are gain-of-function mutations responsible for the alteration of cell proliferation and migration via impairment of MT polymerization using a neuronal model (i.e., SH-SY5Y cells). These results are in agreement with the homodimeric form of Prune_1 as reported in vitro (38) and confirmed in cells (41).

In contrast, another study reported that the missense c.G316A (p.D106N) and c.G383A (p.R128Q) variants result in a complete loss of PPX/PPase enzymatic activity on both P3 and P4 as substrates with respect to the wild-type Prune_1 protein, thus suggesting a loss-of-function activity for these mutants (54). Additionally, in the same paper, the authors showed an increased proteasome-dependent intracellular degradation of mutant p.D30N and p.D106N Prune_1 proteins using unsynchronized human embryonic kidney 293 (HEK-293) cells. In contrast, cells expressing the mutant p.G174* protein showed absence of Prune_1, while those expressing mutant p.R128Q resulted in an amount of Prune_1 protein comparable to that expressed by wild-type HEK-293 cells (54). Furthermore, homozygous null alleles in Prune_1 knockout mice were embryonic lethal at E9.5 with several vascular anomalies, including those in the yolk sac and in the cephalic vascular system (54). Thus, in contrast to the previous study, these data suggest a reduced function of mutant Prune_1 proteins due to hypomorphic variant alleles. Future research studies should be focused on this topic to functionally dissect these "gain or loss" single amino acid changes in PRUNE_1 locus using in vivo state-of-the-art mouse development technology and/or functional analyses in fibroblast-derived organoids from affected patients.

\section{PRUNE_1 IN TUMORIGENESIS}

To date, high expressions of Prune_1 have been found in several metastatic solid tumors: MB groups 3 and 4 (11), gastric cancer (GC) (55), esophageal squamous cell carcinoma (ESCC) (56), NSCLC (8), thyroid cancer (TC) (57), colorectal cancer (CRC) 
(58), NBL (37), BC, and metastatic triple-negative breast cancer (TNBC) $(6,45,59)$. Prune_1 overexpression occurs via the amplification and/or gain of chromosome 1q (as identified in tumors of epithelial origin), where PRUNE_1 is located (i.e., 1q21.3) (6). For instance, MB group 3 ( $\gamma$-subtype), in which Prune_1 was found overexpressed, has a trend for gain of chromosome 1q (60). Moreover, the gain of chromosomal region 1q21 was predominantly reported in $\mathrm{BC}$ belonging to the "basal-like" and TNBC subgroups (30\%-40\%) (61) and also in those recurrent (70\%) (62), in which the levels of Prune_1 were found higher (45).

\section{MEDULLOBLASTOMA}

$\mathrm{MB}$ is an embryonal tumor occurring in the cerebellum and represents $\sim 20 \%$ of all primary childhood CNS tumors. Recent integrative genomics, messenger RNA (mRNA) expression, and methylation profiling have allowed $\mathrm{MB}$ to be stratified into different molecular subgroups: Wingless (WNT), Sonic Hedgehog (SHH), group 3, and group 4 (60). Heterogeneity within these subgroups has been recognized, and it has been suggested that MB may consist of up 12 subtypes [i.e., WNT ( $\alpha$ and $\beta)$, SHH ( $\alpha, \beta, \gamma$, and $\delta$ ), group $3(\alpha, \beta$, and $\gamma)$, and group 4 $(\alpha, \beta$, and $\gamma)]$ (60). Distinctive transcriptional, mutational, and epigenetic profiles were reported for each $\mathrm{MB}$ subtype, with different clinical features (60). The MB SHH subgroup is characterized by an aberrant activation of the SHH pathway and arises from cerebellar granule neuron progenitors (GNPs) mainly mutated in PTCH1 or SMO receptors, although a noncanonical SHH/GLI activation has also been frequently observed (60). Although $\sim 60 \%$ of $\mathrm{MB}$ belongs to groups 3 and 4 , future studies are needed to clarify the developmental origins and the biological mechanisms of these latest subgroups (60). MB group 3 is considered the most aggressive subgroup because of the high metastatic potential and the poor survival rate. The amplification of c-MYC is a common genetic feature in $\mathrm{MB}$ group 3 patients that was found to be inversely correlated with the clinical outcomes (63). One-third of MB group 4 patients are diagnosed with metastasis and show recurrent alterations in the genes involved in chromatin modification (64). Furthermore, amplifications of the MYCN and cyclin-dependent kinase 6 (CDK6) genes are alterations more commonly found in $\mathrm{MB}$ group 4 (65). In contrast, isochromosome $17 \mathrm{q}$ is found in more than $50 \%$ of both MB group 3 and 4 patients, thus being considered a common cytogenetic hallmark for these two subgroups. Of interest is that bulk high-throughput genomic profiling studies have recently reported a strong heterogeneity in $\mathrm{MB}$ groups 3 and 4 in terms of molecular and clinical features, thus showing a subset of tumors with overlapping signatures (66). The impact of this heterogeneity on therapy has been limited to trials testing Smoothened (SMO) antagonists for patients with $\mathrm{MB} \mathrm{SHH} \mathrm{(67)} \mathrm{and} \mathrm{efforts} \mathrm{to}$ reduce therapy for those children affected by the MB WNT subgroup (68), who have relatively good outcomes. Outside of these trials, tailored therapies for $\mathrm{MB}$ groups 3 and 4 are currently lacking, and the combination of surgery, craniospinal radiotherapy (except in young children for whom radiotherapy has devastating neurocognitive side effects), and multi-agent chemotherapy is used (69).

Recently, high expressions of Prune_1 were found in these metastatic MB subgroups (i.e., MB groups 3 and 4), and a new metastatic axis (independent of c-MYC amplification) was dissected in $\mathrm{MB}$ group 3 with the poorest prognosis. In this regard, Prune_1 protein, due to the binding to Nm23-H1, enhances the canonical TGF- $\beta$ pathway, thus leading to OTX2 and SNAIL upregulation, PTEN reduction, and EMT activation (11). Furthermore, gene expression and gene ontology analyses allowed other genes (i.e., OTX2, CYFIP1, and GLI2) involved in neurogenesis to be correlated to Prune_1 (11). Interestingly, a cell competitive permeable peptide (cell-penetrating peptide, $\mathrm{CPP}$ ) that impairs Prune_1/Nn23-H1 complex formation was found to impair both tumorigenesis and metastatic spread in orthotopic models implanted with metastatic MB group 3 cells overexpressing c-MYC and mutated for TP53 (i.e., D425-Med cells), thus representing the most aggressive type of $\mathrm{MB}$ with the poorest prognosis (70). Furthermore, an anti-Prune_1 molecule (pyrimido-pyrimidine derivative, i.e., AA7.1) (23) was also found with the ability to bind Prune_1 protein, to enhance its intracellular degradation and to increase the PTEN protein level, thus inhibiting the Prune_1-mediated metastatic network both in vitro in primary human medullospheres obtained from patients and also in vivo in orthotopic xenograft models of metastatic MB group 3 (11).

\section{Other Tumours}

\section{Gastric Cancer}

Prune_1 was found overexpressed in one-third of a cohort of human GC as measured via quantitative PCR (qPCR) and immunohistochemical analyses (55). Prune_1 was positively correlated with advanced tumor grade, lymph node metastasis, and poor prognosis. In this regard, GC patients expressing Prune_1 showed worse survival rates compared to those in which Prune_1 was not detected. Furthermore, the majority of these GC specimens (87\%) were also found with positivity for the $\mathrm{Nm} 23-\mathrm{H} 1$ protein. Interestingly, the presence of $\mathrm{Nm} 23-\mathrm{H} 1$ was detected in all Prune_1-positive GC samples. Altogether, these findings indicate that the overexpression of both Prune_1 and $\mathrm{Nm} 23-\mathrm{H} 1$ is associated with poor clinical outcomes in GC (55).

\section{Esophageal Squamous Cell Carcinoma}

Prune_1 was also found increased in $21 \%$ of a cohort $(n=205)$ of ESCC patients. Its protein levels were found to be positively correlated with the tumor size (T; $p<0.0001)$ and regional lymph nodes $(\mathrm{N} ; p<0.0001)$. These data show that PRUNE_1 could be used to identify ESCC patients with increased risk of disease recurrence or poor clinical outcomes (56).

\section{Non-Small Cell Lung Cancer}

Prune_1 was shown to modulate the Wnt pathway in NSCLC via its binding to GSK-3 $\beta$ and, as a consequence, the activation of $\beta$-catenin cascade (8). In this regard, Prune_1 silencing 
(through adenoviral approach) resulted in the reduction of activated $\beta$-catenin, inhibition of cell invasion, and decrease of the proliferation rate in NSCLC cells (8). Furthermore, the silencing of Prune_1 was also reported to inhibit lung metastasis formation in vivo using a murine xenograft model injected ( $v i a$ tail vein) with A549-Luc cells (overexpressing the firefly luciferase gene) silenced for Prune_1. Tumorigenesis was followed using in vivo bioluminescence (BLI) technology (71). Mice receiving these cells silenced for Prune_1 showed significant reduction of the tumor burden and lung metastasis nodules compared to those in the control group (8). Furthermore, Prune_1 protein was also detected in the serum of NSCLC patients via ELISA (8), thus suggesting that it could represent an early diagnostic marker for this type of tumors.

\section{Thyroid Cancer}

The role of Prune_1 in TC was also studied (57). In this regard, Prune_1 was detected in anaplastic TC and metastatic lymph node specimens. Prune_1 silencing and the inhibition of its PDE activity via dipyridamole were found to inhibit cell motility in human TC cells (i.e., 8505C and KTC-3) (57). These data were also confirmed in vivo, in which the inhibition of Prune_1 was reported to suppress tumor invasion and pulmonary metastasis in an orthotopic mouse model of TC (57). Thus, these analyses altogether confirm Prune_1 as a candidate target in anaplastic TC.

\section{Colorectal Cancer}

Prune_1 expression was found to be correlated with cell motility and EMT in CRC liver metastases (CRLM) (58). Prune_1 was found to positively regulate migration and EMT processes through in vitro assays performed using cells overexpressing or silenced for Prune_1 (58). Furthermore, in vivo experiments (with murine xenograft models) also confirmed the association of Prune_1 with tumor invasion and distant metastases (58). Interestingly, immunohistochemical analyses detected the Prune_1 protein in $28 \%$ of a cohort of CRC patients. Importantly, these Prune_1-positive CRC tumors showed significant lower overall survival (OS) rates $(p=$ 0.003) (58).

\section{Hepatocellular Carcinoma}

Prune_1 was found overexpressed in HCC in a cohort of 304 patients using tissue microarray (TMA) (72). Of importance is that the expression of Prune_1 was found to be correlated with poorer OS and disease-free survival (DFS). Furthermore, gene expression analyses performed in HCC patients overexpressing Prune_1 demonstrated a statistically significant enrichment of the genes with roles in proliferative processes, DNA methylation, and the canonical Wnt pathway (72). Moreover, mutational spectra in HCC tumors with higher Prune_1 protein levels showed higher mutation burdens in RPS6KA3 and RB1 genes (72). Altogether, these findings suggest that PRUNE_1 acts as a tumor promoter gene in HCC. Furthermore, at this time, its role in the epigenetic changes occurring in cancer-related genes can be postulated.

\section{Breast Cancer and Triple-Negative Breast Cancer}

High expression levels of Prune_1 were found associated with metastasis in regional lymph nodes $(p=0.017)$ and in distant sites $(p=0.029)$ in a cohort of BC-affected patients (45). These results were also recently confirmed in patients with TNBC through TMA immunohistochemistry technologies (59). In detail, the levels of Prune_1 protein had a positive correlation with distant metastasis (lung) and infiltrating pro-tumorigenic tumor-associated macrophages (M2-TAMs) with antiinflammatory functions in the tumor microenvironment (TME). Furthermore, a genetically engineered mouse model (GEMM) of metastatic TNBC characterized by the overexpression of both human Prune_1 and Wnt1 in breast [through the use of the mouse mammary tumour virus (MMTV) promoter, i.e., MMTV-Prune_1/Wnt1] revealed that Prune_1 enhances the polarization of TAMs toward the M2 phenotype in the TME via the activation of the canonical (SMAD2-mediated) TGF- $\beta$ pathway, IL17F secretion, and extracellular vesicle protein content modulation (59). Worth noting is that the non-toxic small anti-Prune_1 molecule (i.e., AA7.1) was found with the ability to impair the extracellular crosstalk between TNBC cells characterized by Prune_1 overexpression and TAMs, thus reducing the metastatic properties of the tumorigenic cells in vivo by using a GEMM of metastatic TNBC (i.e., MMTVPrune_1/Wnt1) (59).

\section{Neuroblastoma}

Prune_1 and its interactor Nm23-H1 were also found significantly overexpressed in NBL (37). Interestingly, a positive correlation trend between Nm23-H1, Prune_1, and patients' survival rates was also reported (37). Taken altogether, these findings suggest that the formation of the Prune_1/Nm23-H1 complex may have a role in cancer progression in NBL. Furthermore, high levels of Prune_1 and $\mathrm{Nm} 23-\mathrm{H} 1$ increased the aggressiveness of NBL cells (SH-SY5Y and SK-N-BE) in vitro and in vivo (37). In this regard, Prune_1 and $\mathrm{Nm} 23-\mathrm{H} 1$ were found to enhance the formation of metastatic foci in a mouse orthotopic xenograft model of NBL (37). Importantly, a cell CPP mimicking the region of Nm23-H1 that is responsible for its interaction with Prune_1 was found with the ability to impair the cell motility in vitro and the tumor growth and metastasis formation in vivo (37). Thus, the Prune_1/Nm23-H1 complex was found to enhance NBL tumorigenesis, and its impairment using CPP may be a useful strategy for NBL treatment.

\section{PRUNE_1 AS TARGET FOR ANTI- TUMORIGENIC THERAPIES}

\section{Targeting Prune_1/Nm23-H1 Complex}

Due to their prominent role in metastatic tumors, targeting the Prune_1/Nm23-H1 protein complex represents a promising therapeutic target for cancer treatment. The protein complex formation between Prune_1 and Nm23-H1 is dependent on casein kinase 1 (CKI)-mediated 
TABLE 2 | List of the therapeutic strategies to impair Prune_1 in vitro and/or in vivo.

\begin{tabular}{|c|c|c|c|c|}
\hline Tumour type & $\begin{array}{l}\text { Treatment } \\
\text { strategy }\end{array}$ & In vitro (cell lines) & In vivo (murine models) & References \\
\hline $\begin{array}{l}\text { Medulloblastoma } \\
(\mathrm{MB})\end{array}$ & $\begin{array}{l}\text { AA7.1 } \\
\text { CPP }\end{array}$ & $\begin{array}{l}\text { AA7.1: MB group } 3 \text { cell lines (D283-MED, D341-MED, } \\
\text { D425-MED) and primary MB group3/4 cells } \\
\text { CPP: MB group } 3 \text { cell lines (D283-MED and D425-MED) }\end{array}$ & $\begin{array}{l}\text { AA7.1 and CPP: orthotopic xenograft mouse models } \\
\text { with metastatic MB group } 3 \text { cell line (D425-MED) }\end{array}$ & $(11)$ \\
\hline $\begin{array}{l}\text { Neuroblastoma } \\
\text { (NBL) }\end{array}$ & CPP & NBL cell line (SH-SY5Y) & $\begin{array}{l}\text { Mouse orthotopic xenograft models with NBL cells (SH- } \\
\text { SY5Y) }\end{array}$ & $(8,73)$ \\
\hline $\begin{array}{l}\text { Triple-negative } \\
\text { breast cancer } \\
\text { (TNBC) }\end{array}$ & $\begin{array}{l}\text { AA7.1 } \\
\text { Dipyridamole } \\
\text { CPP }\end{array}$ & $\begin{array}{l}\text { AA7.1: murine TNBC cells (MMTV-Prune_1Mnt1) } \\
\text { Dipyridamole: murine and human TNBC cell lines (4T1 } \\
\text { and MDA-MB-231T) } \\
\text { CPP: TNBC cell lines (MDA-MB-231T) }\end{array}$ & $\begin{array}{l}\text { AA7.1: GEMM of metastatic TNBC (MMTV-Prune_1/ } \\
\text { Wnt1 cells) } \\
\text { Dipyridamole: orthotopic xenograft mouse models with } \\
\text { TNBC cell lines (MDA-MB-231T and } 4 T 1 \text { ) }\end{array}$ & $(22,59,73)$ \\
\hline Breast cancer (BC) & CPP & $\mathrm{BC}(\mathrm{ER}+, \mathrm{PG}+)$ cell line (MCF-7) & & (73) \\
\hline Thyroid cancer (TC) & Dipyridamole & & $\begin{array}{l}\text { Orthotopic xenograft mouse models with TC cell line } \\
\text { (8505C) }\end{array}$ & $(57)$ \\
\hline Prostate cancer & CPP & Prostate cancer cell line (PC3) & $\begin{array}{l}\text { Orthotopic xenograft mouse models with prostate } \\
\text { cancer cell line (PC3) }\end{array}$ & (73) \\
\hline $\begin{array}{l}\text { Colorectal cancer } \\
(\mathrm{CRC})\end{array}$ & CPP & CRC cell lines (HT29 and SW480) & & (73) \\
\hline
\end{tabular}

GEMM, genetically engineered mouse model; CPP, cell-penetrating peptide.

phosphorylation in S120, S122, and S125 on the C-terminus domain of Nm23-H1 protein (41). Thus, therapeutic strategies aimed to inhibit Prune_1/Nm23-H1 complex formation via targeting these phosphorylation processes could be applied in tumorigenic cells. In this regard, a CPP was developed to mimic the region of Nm23-H1 (from amino acids 115 to 129) that contains these residues (i.e., S120, S122, and S125) that are phosphorylated by the CKI enzyme. The ability of CPP to impair the Prune_1/Nm23-H1 complex was assayed in different tumorigenic cells in vitro (Table 2) (73). As a consequence, CPP reduced the cell proliferation and cell motility in vitro in $\mathrm{BC}$, prostate cancer (PC), $\mathrm{CRC}, \mathrm{NBL}$, and MB cells $(11,73)$. The delivery of CPP in these tumorigenic cells was performed through adenoviral particles carrying the sequences encoding for CPP and for the transactivating protein of HIV. The absence of cytotoxicity in vitro in nontumorigenic cells (i.e., HEK-293) indicated a specific action of CPP against cancer cells. The same adenoviral approach was also used in vivo (using xenograft murine models) to show the therapeutic benefits of CPP against PC, NBL, and $\mathrm{MB}$ (Table 2) (11, 37, 73). Of importance is that the biosafety of $\mathrm{CPP}$ in mice was also reported in terms of hematological parameters. Altogether, these data address the potential future use of CPP for the treatment of PC, NBL, and MB.

\section{Targeting Prune_1 Enzymatic Activities}

The impairment of the PDE or PPX/PPase activities of Prune_1 represents another promising anti-tumorigenic strategy to impair tumor progression. In this regard, the PDE activity of Prune_1 was found to stimulate cellular motility in vitro and, as a consequence, metastatic progression. Furthermore, the antitumorigenic action of dipyridamole (an anti-platelet aggregation agent and one of the selective PDE inhibitors) in TNBC was also investigated in vivo using xenograft mice (Table 2) (22). Thus, PDE inhibitors could be used as therapeutic agents for Prune_1-overexpressing tumors. Interestingly, dipyridamole did not affect the PPX/PPase enzymatic function of Prune_1, as measured using P3 as a substrate (7).

The PPX/PPAse activity was shown to be diminished by $\mathrm{Nm} 23-\mathrm{H} 1$ in vitro (7). Indeed, Nm23-H1 was able to inhibit the P3-hydrolyzing activity of Prune_1 at micromolar concentrations (7). These data suggest a potential binding competition between $\mathrm{Nm} 23-\mathrm{H} 1$ and the P3 substrate for Prune_1. Thus, small-molecule activators of Nm23-H1 (e.g., NMac1) (74) represent future strategies to impair cancer progression in tumors characterized by the overexpression of Prune_1 and Nm23-H1.

It is of interest that long-chain polyPs (poor substrates of Prune_1) were also found to reduce the Prune_1-catalyzed hydrolysis of P3 substrate (7). This inhibitory effect may be due to $\mathrm{Mg}^{2+}$ chelation or to the competition between P3 and long-chain polyPs for active site binding of Prune_1. To date, other studies have reported on the anti-tumorigenic actions of polyPs. In this regard, the anti-metastatic activity of polyPs (P75) was reported for melanoma in vivo using murine xenograft models (75). Furthermore, treatment with polyPs (P120) was found to reduce the intracellular ATP level in NSCLC and increased their sensitivity to X-irradiation (76). Thus, polyPs can be promising targets for the development of novel antitumorigenic therapies in humans.

\section{Targeting Prune_1 as Downregulating Its Protein Levels}

To date, a pyrimido-pyrimidine derivative (AA7.1) was found with the ability to decrease Prune_1 protein intracellular levels may be via enhancement of its proteasomal-dependent degradation (11). Protein-drug interaction studies (via NMR approaches) showed the amino acid residues of Prune_1 that are mainly responsible for the binding to AA7.1 (i.e., L359 and D364) (11). This molecule was also found able to decrease Prune_1 mRNA and protein levels in different MB group 3 (11) and TNBC (59) cells (Table 2). In vitro and in vivo assays also showed the ability of AA7.1 to decrease the cell proliferative 
and migratory processes (Table 2). In detail, in MB group 3, AA7.1 impaired the metastatic axis driven by Prune_1, thus leading to impairment in TGF- $\beta$, decreased levels of OTX2, upregulation of PTEN, inhibition of EMT, reduction in Nestin, and increases in Tuj1 and GFAP differentiation neuronal markers (11). These results suggest the potential of AA7.1 to inhibit the neural stem/progenitor cell markers and to increase neuronal differentiation processes, thus inhibiting the role of Prune_1 in these very important biological processes during neurogenesis.

Furthermore, the pharmacological inhibition of Prune_1 protein in TNBC (achieved through AA7.1 treatment) decreased the number of metastatic foci in vivo also via inhibiting the switch of TAMs in the TME toward the M2 phenotype (59). In TNBC, AA7.1-mediated tumor inhibition occurred through the impairment of the TGF- $\beta$ pathway, reduction of inflammatory cytokines (i.e., IL-17F) and the modulation of the protein content of extracellular vesicles (i.e., vimentin) (59). Importantly, this small molecule is not toxic, as suggested by the lack of acute toxicity measured in naive mice $(\mathrm{Balb} / \mathrm{C})$ that were intraperitoneally administered escalating doses $(15,30$, and $60 \mathrm{mg} / \mathrm{kg})$ of AA7.1 daily for 1 week (11). The results showed no immediate acute toxicity of AA7.1 (in terms of hematological parameters, hepatotoxicity, or nephrotoxicity) in treated mice, as measured via glutamate-pyruvate transaminase 1, glutamic oxaloacetic transaminase, creatinine blood levels, and blood urea nitrogen (11). Thus, altogether, these findings suggest that AA7.1 is a nontoxic potential immunomodulatory molecule with the ability to modulate the inflammatory processes in the TME, thus inhibiting the metastatic spread in Prune_1-overexpressing tumors.

To date, none of these therapeutic approaches (as summarized in Table 2) has entered clinical testing. However, the pharmacological approach via Prune_1 downregulation (though AA7.1 treatment) has shown therapeutic benefits in murine models of $\mathrm{MB}$ and TNBC with the absence of acute toxicity (11). Regarding the inhibition of Prune_1/Nm23-H1 complex formation, CPP was also tested in vivo in xenograft mice of NBL and prostate cancer, thus also representing a promising strategy against tumors overexpressing both Prune_1 and Nm23-H1. Therefore, future studies should be focused on developing i) small molecules (e.g., AA7.1 derivatives) with a higher affinity to Prune_1 in order to reduce the dose to be used in vivo and ii) synthetic peptides with the ability to impair the interaction between Prune_1 and Nm23-H1, hence overcoming the use of adenoviral infection that might suffer from tissuespecific trophism.

\section{DISCUSSION}

Neural development involves a dynamic orchestrated sequence of cellular events driven by proliferation and migration processes involving neural progenitor cells (77). A tight spatial/temporal regulation of the proliferation and migration events in neural progenitor cells (NPCs) may prevent developmental malformations (as a result of hypo-proliferation) and tumor formation (due to hyper-proliferation). In this regard, NDDs (e.g., MCPH) may be caused by genetic mutations affecting the genes that modulate the proliferation and migration properties of NPCs. Similarly, brain tumorigenic cells can also derive from NPCs. Thus, the proliferation rate of brain tumorigenic cells may be regulated by the same genes that exert control on normal proliferative cells (78). Indeed, the different MB subgroups can originate from neural stem cells residing in the $\mathrm{VZ}$ of the cerebellum or from NPCs localized in the external granular layer of the cerebellum or rhombic lip (79). Since MB cells have been found to retain the genetic and molecular features of the cell of origin (80), the proteins involved in the growth and survival processes of neural stem cells or NPCs may also exert the same functions in brain tumorigenic cells.

Among these proteins, the PRUNE_1 gene was reported both mutated in NDDs (i.e., NMIHBA) and overexpressed in pediatric brain tumors (i.e., MB). In this regard, Prune_1 was found with a fundamental role in the proliferation and migration processes occurring during neural development. Its expression, together with $\mathrm{Nm} 23-\mathrm{H} 1$, was detected in the mouse developing brain in the cortex, hippocampus, midbrain, and in the VZ, in which proliferation processes take place (5). Furthermore, mutations in the different regions of the human PRUNE_1 gene (mainly in the region encoding for the $\mathrm{DHH}$ domain) were found to be causative of NDDs characterized by MCPH (the majority of the patients) and other brain anomalies (i.e., NMIHBA). At the same time, the Prune_1 protein was also described as overexpressed in both CNS and PNS tumors, including pediatric $\mathrm{MB}$ group 3, which drives the metastatic spread (11).

Thus, the deregulation of Prune_1 in somatic or germinal cells may result in oncogenesis or neural developmental defects, respectively. The explanation for this dual phenotypic effect might be provided by the pleiotropic role of Prune_1 in modulating several cellular processes, including proliferation, migration, MT polymerization during mitosis, and the regulation of signaling pathways.

We think that all these different functions are modulated by the enzymatic activities of Prune_1 (i.e., PPX/PPase and phosphodiesterase) and its property of being a naturally unfolded protein able to interact with other proteins with a role in these processes (i.e., Nm-23-H1/H2 and GSK-3 $\beta$ ) (Figure 1C). How these interactions influence these processes is still to be discovered in detail in the near future. All these different functions are regulated by the enzymatic activities of Prune_1 (i.e., PPX/PPase and phosphodiesterase) and its unfolded structure in the C-terminus domain that allows it to interact with different proteins (i.e., Nm-23-H1/H2 and GSK3ß) (Figure 1C).

Of interest is that, through in silico analyses of 5,000 bp upstream of the transcription start site (TSS) of the PRUNE_1 gene, a number of sequences predicted to act as binding sites for transcriptional regulators have been identified (see Figures 2A-D and Table 3). This genomic region also contains sites (highly 
A

$\operatorname{chr} 1: 151,003,425-151,035,704$
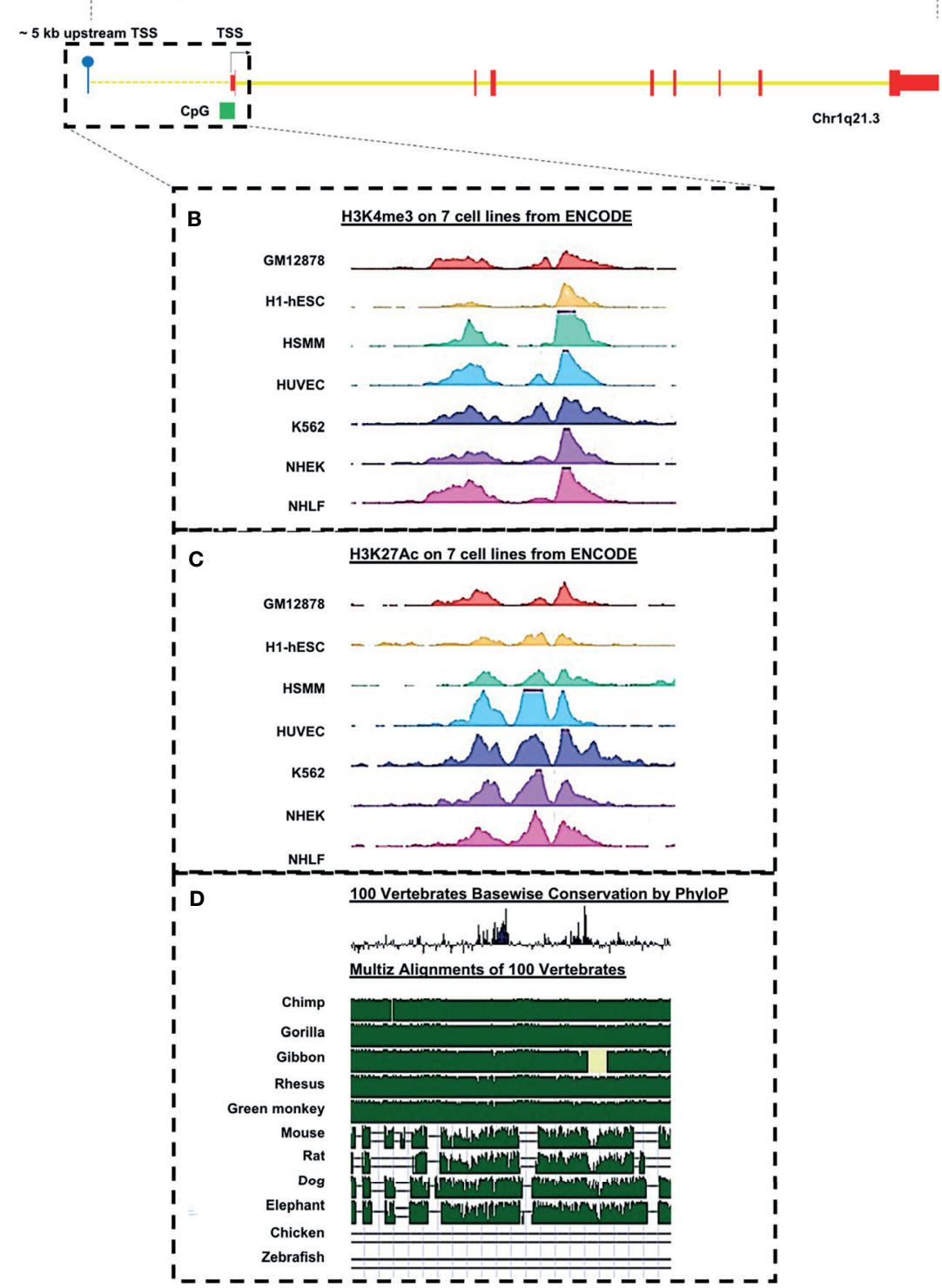

FIGURE 2 | Representation of putative transcriptional factors predicted to bind the regulatory regions of the PRUNE_1 gene. (A) Schematic representation of PRUNE_1 gene and its upstream putative regulatory region as reported in the UCSC Genome Browser on Human Dec. 2013 (GRCh38/hg38) Assembly (https:// genome.ucsc.edu/). The first exon (in red) is composed of a 5'UTR and a coding region. Introns are depicted in yellow. A CpG island (in green) is found in the promoter region of the gene. (B) The upstream region of PRUNE_1 shows H3K4me3 peaks, as marker of the promoter region. (C) The upstream region of PRUNE_1 shows H3K27Ac peaks, as marker of the enhancer regions. (B, C) Data obtained from chromatin immunoprecipitation sequencing (ChIP-seq) experiments performed on seven different cell lines, as reported in ENCODE (https://www.encodeproject.org/). (D) Peaks of the conserved genomic elements upon species are present in the upstream region of the gene.

conserved through evolution) targeted by epigenetic modifications. Furthermore, the presence of a $\mathrm{CpG}$ island was also found (Figure 2A), thus suggesting potential DNA methylation sites associated with transcriptional repression. Overall, these predicted sites could be of importance for potential negative/positive modulation of PRUNE_1 expression. Of note is that, among these putative transcriptional factors present at the $5^{\prime}$ region of the PRUNE_1 gene (Table 3), we found mutations in SMARCA4 that are available in a significant frequency of $\mathrm{MB}$ and pancreatic cancers and in many other tumor subtypes (81-83). SMARCA4 was reported as the most frequently mutated gene in MB group 3 (83). Future research issues will be aimed at defining this finding and the transcriptional regulation of the PRUNE_1 gene program in neurogenesis, development, 
TABLE 3 | List of putative transcription factor predicted to bind the regulatory elements (ORegAnno https://www.bcgsc.ca/resources/software/oreganno) 5000 bp upstream of the transcription start site of PRUNE_1 gene.

\begin{tabular}{|c|c|c|c|}
\hline $\begin{array}{l}\text { Transcription } \\
\text { factor }\end{array}$ & Chromosomal position (hg38 assembly) & Consensus sequences & Biological process \\
\hline CTCF & chr1:151006584-151007134; chr1:151006744-151007094 & 5'-CCGCGNGGNGGCAG-3' & Development \\
\hline FOXA1 & chr1:151007690-151008630 & $\begin{array}{l}5^{\prime}-[\mathrm{AC}] \mathrm{A}[\mathrm{AT}] \mathrm{T}[\mathrm{AG}] \mathrm{TT}[\mathrm{GT}][\mathrm{AG}][\mathrm{CT}] \mathrm{T}[\mathrm{CT}]- \\
3^{\prime}\end{array}$ & Development \\
\hline$R B L 2$ & $\begin{array}{l}\text { chr1:151006386-151006759; chr1:151007954-151008902; } \\
\text { chr1:151007961-151008350 }\end{array}$ & $\begin{array}{l}\text { Co-repressor (not directly binding to } \\
\text { DNA) }\end{array}$ & $\begin{array}{l}\text { Cell division/neuronal } \\
\text { differentiation }\end{array}$ \\
\hline SMARCA4 & chr1:151004300-151009699 & $\begin{array}{l}\text { SWI/SNF subunit (chromatin remodeling } \\
\text { complex) }\end{array}$ & Neuronal differentiation \\
\hline TFAP2C & chr1:151007930-151008730 & $5^{\prime}-$ GCCNNNGGC-3' & Neural tube development \\
\hline
\end{tabular}

epidermal differentiation, and cell cycle regulation. However, the roles and the transcriptional mechanisms of Prune_1 in both oncogenesis and NDDs still need further future investigations.

The mechanisms responsible for correct mitosis are of importance for both normal brain development and primary brain tumor growth (i.e., MB). Besides PRUNE_1, the other five genes (i.e., KIF11, KIF14, ASPM, CDK6, and ATR) have been reported as mutated in hereditary $\mathrm{MCPH}$ and overexpressed in primary brain tumors (e.g., MB). The products encoded by these genes are known to play crucial roles in regulating mitotic entry or cell cycle progression (78). Here, we analyzed public gene expression data related to the genes reported as causative of primary $\mathrm{MCPH}(47,48)$ (Table 4) and PRUNE_1 by interrogating available $\mathrm{MB}$ datasets (i.e., Pfister, Delattre, Gilbertson, and Kool) through the R2 Genomics Analysis and Visualization Platform (http://r2.amc.nl) (Figure 3). Interestingly, these data showed that the expression levels of the majority of these genes (i.e., 77.8\%) were higher in $\mathrm{MB}$ samples compared to those in normal cerebellum (Figure 3). On these genes mutated in $\mathrm{MCPH}$ and overexpressed in $\mathrm{MB}$, further analyses were performed using KEGG in order to identify a potential network of proteins (Figure 4). The data showed that these proteins are mainly involved in biological processes such as cell cycle (red, GO:0007049), mitotic cell cycle (blue, GO:1903047), MT-based process (green, GO:0007017), and CNS development (yellow, GO:0007417). Future studies will be needed to study the transcriptional activation of these genes and their functions on proliferation and migration in brain tumors.

Intriguingly, Prune_1 was reported to activate the canonical Wnt pathway through its binding to GSK-3 $\beta$. However, its overexpression in $\mathrm{MB}$ was found in metastatic groups 3 and 4, but not in tumors belonging to the Wnt molecular subgroup. This may be due to the different frequencies of genetic aberration (e.g., amplification of chromosome 1q) in the different subtypes of MB (60) and also because of the heterogeneity among the MB subtypes and their distinct developmental origins. In this regard, the cerebellum is thought to be the origin site for $\mathrm{MB} \mathrm{SHH}$, group 3 , and group 4, while the lower rhombic lip of the developing brain stem seems to represent the site from which MB WNT arises (79). More in detail, the cells of origin for $\mathrm{MB}$ SHH are the granule cell precursors (GCPs) (79). In contrast, MB group 3 develops from Nestin $^{+}$cerebellar stem cells, and MB group 4 derives from progenitor cells giving rise to both GCP and unipolar brush cell (UBC) lineages (79).

To date, among the mutations identified in PRUNE_1 locus in NMIHBA patients, only three gene variants have been reported within the public data of Catalog of Somatic Mutations in Cancer (COSMIC, v94, released May 29, 2021) of 39,615 samples collected. In detail, the missense p.D30N Prune_1 variant (COSM5843581) was found in one patient affected with malignant melanoma, the p.R297W mutation (COSM462922) was reported in one subject suffering from clear cell renal cell carcinoma and in three patients diagnosed with colon adenocarcinoma, and the splicing c.521-2A $>$ G variant (COSM7836151) was found in one patient with squamous cell lung carcinoma. Nevertheless, because of the paucity of tumor samples with PRUNE_1 mutations and due to the lack of information regarding the zygosity of the identified variants, we cannot hypothesize a role for these PRUNE_1 mutations in tumorigenic processes. Thus, further studies will be needed to address this issue.

Different genotype to phenotype correlation studies have been reported to dissect the role of mutant Prune_1 protein in children affected with NMIHBA. In this regard, opposing enzymatic activities for mutated Prune_1 proteins have been reported $(10,54)$. The contrasting results about the biochemical activity of the mutant Prune_1 proteins could be reasoned by the homodimeric form reported for Prune_1 (38), the interaction with intracellular binding partners (whose protein abundance is dependent of the cell district) that can modulate its enzymatic function (e.g., Nm23-H1) (7), and the possible oscillatory changes in Prune_1 protein abundance (and activity) during the cell cycle because of its role in the mitotic spindle during mitosis (10). Thus, further studies taking into account the potential role of polyPs at short chain lengths (as Prune_1 substrates) in neurogenesis and the homodimeric structure of Prune_1 and its interactors will be needed to address this issue.

Of interest is that Prune_1 acts as a MAP, enhances the MT polymerization (in vitro and in cells), and co-localizes with $\beta$ tubulin in the mitotic spindle in dividing cells (10). Importantly, its interactor, $\mathrm{Nm} 23-\mathrm{H} 1$, was also described to be an active constituent of the centrosomes (84) and was reported to be associated with $\alpha$ - and $\beta$-tubulins with a role in their 
TABLE 4 | List of the genes identified as causative for primary microcephaly (MCPH).

Gene
name

MCPH1 Microcephalin. Implicated in chromosome condensation and DNA damage-induced cellular responses. May play a role in neurogenesis and regulation of the size of the cerebral cortex.

CDK5RAP2 CDK5 regulatory subunit-associated protein 2. Potential regulator of CDK5 activity via its interaction with CDK5R1. Negative regulator of centriole disengagement (licensing), which maintains centriole engagement and cohesion. Involved in the regulation of mitotic spindle orientation. Plays a role in spindle checkpoint activation by acting as a transcriptional regulator of both BUBR1 and MAD2 promoters. Together with MAPRE1, it may promote microtubule polymerization, bundle formation, growth, and dynamics at the plus ends. Regulates centrosomal maturation.

CEP152 Centrosomal protein of $152 \mathrm{kDa}$. Necessary for centrosome duplication; the function seems to also involve CEP63, CDK5RAP2, and WDR62 through a stepwise assembled complex at the centrosome that recruits CDK2 required for centriole duplication. Acts as a molecular scaffold facilitating the interaction of PLK4 and CENPJ, two molecules involved in centriole formation. Proposed to snatch PLK4 away from PLK4:CEP92 complexes in early G1 daughter centriole and to reposition PLK4 at the outer boundary of a newly forming CEP152 ring structure. Also plays a key role in deuterosome-mediated centriole.

ASPM Abnormal spindle-like microcephaly-associated protein. Involved in mitotic spindle regulation and coordination of mitotic processes. Its function in regulating microtubule dynamics at spindle poles, including spindle orientation, astral microtubule density, and poleward microtubule flux, seems to depend on the association with the katanin complex formed by KATNA1 and KATNB1. Enhances the microtubule lattice severing activity of KATNA1 by recruiting the katanin complex to microtubules. Can block microtubule minus-end growth; reversely, this function can be enhanced by the katanin complex.

CENPJ Centromere protein J. Plays an important role in cell division and centrosome function by participating in centriole duplication. Inhibits microtubule nucleation from the centrosome. Involved in the regulation of slow processive growth of centriolar microtubules. Acts as a microtubule plus-end tracking protein that stabilizes centriolar microtubules and inhibits microtubule polymerization and extension from the distal ends of centrioles. Required for centriole elongation and for STIL-mediated centriole amplification. May be involved in the control of centriolar microtubule growth.

STIL SCL-interrupting locus protein. Immediate-early gene. Plays an important role in embryonic development and in cellular growth and proliferation. Its longterm silencing affects cell survival and cell cycle distribution and decreases CDK1 activity correlated with the reduced phosphorylation of CDK1. Plays a role as a positive regulator of the Sonic Hedgehog pathway, acting downstream of PTCH1. Plays an important role in the regulation of centriole duplication. Required for the onset of procentriole formation and proper mitotic progression.

CEP135 Centrosomal protein of $135 \mathrm{kDa}$. Centrosomal protein involved in centriole biogenesis. Acts as a scaffolding protein during early centriole biogenesis. Required for the targeting of centriole satellite proteins to centrosomes such as of PCM1, SSX2IP, and CEP290 and the recruitment of WRAP73 to centrioles. Also required for centriole-centriole cohesion during interphase by acting as a platform protein for CEP250 at the centriole. Belongs to the CEP135/TSGA10 family.

CASC5 Kinetochore scaffold 1. Performs two crucial functions during mitosis: it is essential for spindle assembly checkpoint signaling and for correct chromosome alignment. Required for the attachment of the kinetochores to the spindle microtubules. Directly links BUB1 and BUB1B to kinetochores. Part of the MIS12 complex, which may be fundamental for kinetochore formation and proper chromosome segregation during mitosis. Acts in coordination with CENPK to recruit the NDC80 complex to the outer kinetochore.

PHC1 Polyhomeotic-like protein 1. Component of a Polycomb group (PcG) multiprotein PRC1-like complex, a complex class required to maintain the transcriptionally repressive state of many genes, including Hox genes, throughout development. PCG PRC1 complex acts via chromatin remodeling and modification of histones. It mediates the monoubiquitination of histone H2A "Lys-119," rendering chromatin heritably changed in its expressibility. Required for proper control of cellular levels of GMNN expression.

CDK6 Cyclin-dependent kinase 6. Serine/threonine protein kinase involved in the control of cell cycle and differentiation. Promotes G1/S transition. Phosphorylates pRB/RB1 and NPM1. Interacts with D-type G1 cyclins during interphase at G1 to form a pRB/RB1 kinase and controls the entrance into the cell cycle. Involved in the initiation and maintenance of cell cycle exit during cell differentiation. Prevents cell proliferation and negatively regulates cell differentiation, but is required for the proliferation of specific cell types (e.g., erythroid and hematopoietic cells).

CENPE Centromere-associated protein E. Microtubule plus-end-directed kinetochore motor that plays an important role in chromosome congression, microtubulekinetochore conjugation, and spindle assembly checkpoint activation. Drives chromosome congression (alignment of chromosomes at the spindle equator resulting in the formation of the metaphase plate) by mediating the lateral sliding of polar chromosomes along spindle microtubules toward the spindle equator and by aiding the establishment and maintenance of connections between kinetochores and spindle microtubules.

ANKLE2 Ankyrin repeat and LEM domain-containing protein 2. Involved in mitotic nuclear envelope reassembly by promoting the dephosphorylation of BAF/BANF1 during mitotic exit. Coordinates the control of BAF/BANF1 dephosphorylation by inhibiting VRK1 kinase and promoting the dephosphorylation of BAF/ BANF1 by protein phosphatase 2A (PP2A), thereby facilitating nuclear envelope assembly. It is unclear whether it acts as a real PP2A regulatory subunit or whether it is involved in the recruitment of the PP2A complex. Involved in brain development.

COPB2 Coatomer subunit beta. The coatomer is a cytosolic protein complex that binds to dilysine motifs and reversibly associates with Golgi non-clathrin-coated vesicles, which further mediate biosynthetic protein transport from the ER via the Golgi up to the trans-Golgi network. The coatomer complex is required for budding from Golgi membranes and is essential for the retrograde Golgi-to-ER transport of dilysine-tagged proteins. In mammals, the coatomer can only be recruited by membranes associated with ADP-ribosylation factors (ARFs), which are small GTP-binding proteins.

KIF14 Kinesin-like protein KIF14. Microtubule motor protein that binds to microtubules with high affinity through each tubulin heterodimer and has an ATPase activity. Plays a role in many processes such as cell division, cytokinesis, and also in cell proliferation and apoptosis. During cytokinesis, targets to the central spindle and midbody through its interaction with PRC1 and CIT, respectively. Regulates cell growth through the regulation of cell cycle progression and cytokinesis. During cell cycle progression, acts through SCF-dependent proteasomal ubiquitin-dependent protein.

NCAPD2 Condensin complex subunit 1. Regulatory subunit of the condensin complex, a complex required for the conversion of interphase chromatin into mitoticlike condense chromosomes. The condensin complex probably introduces positive supercoils into relaxed DNA in the presence of type I topoisomerases and converts nicked DNA into positive knotted forms in the presence of type II topoisomerases. May target the condensin complex to DNA via its Cterminal domain. Belongs to the CND1 (condensin subunit 1) family.

NCAPD3 Condensin-2 complex subunit D3. Regulatory subunit of the condensin-2 complex, a complex that establishes mitotic chromosome architecture and is involved in the physical rigidity of the chromatid axis. 
TABLE 4 | Continued

Gene

name

NCAPH

Condensin complex subunit 2. Regulatory subunit of the condensin complex, a complex required for the conversion of interphase chromatin into mitoticlike condense chromosomes. The condensin complex probably introduces positive supercoils into relaxed DNA in the presence of type I topoisomerases and converts nicked DNA into positive knotted forms in the presence of type II topoisomerases.

NUP37 Nucleoporin Nup37. Component of the Nup107-160 subcomplex of the nuclear pore complex (NPC). The Nup107-160 subcomplex is required for the assembly of a functional NPC. The Nup107-160 subcomplex is also required for normal kinetochore microtubule attachment, mitotic progression, and chromosome segregation.

LMNB1 Lamin-B1. Lamins are components of the nuclear lamina, a fibrous layer on the nucleoplasmic side of the inner nuclear membrane, which is thought to provide a framework for the nuclear envelope and may also interact with chromatin.

LMNB2 Lamin-B2. Lamins are components of the nuclear lamina, a fibrous layer on the nucleoplasmic side of the inner nuclear membrane, which is thought to provide a framework for the nuclear envelope and may also interact with chromatin. Belongs to the intermediate filament family.

WDR62 WD repeat-containing protein 62. Required for cerebral cortical development. Plays a role in neuronal proliferation and migration. Plays a role in mothercentriole-dependent centriole duplication. The function seems to also involve CEP152, CDK5RAP2, and CEP63 through a stepwise assembled complex at the centrosome that recruits CDK2 required for centriole duplication.

MFSD2A Sodium-dependent lysophosphatidylcholine symporter 1. Sodium-dependent lysophosphatidylcholine (LPC) symporter, which plays an essential role for blood-brain barrier formation and function. Specifically expressed in the endothelium of the blood-brain barrier of microvessels and transports LPC into the brain. Transport of LPC is essential because it constitutes the major mechanism by which docosahexaenoic acid (DHA), an omega-3 fatty acid that is essential for normal brain growth and cognitive function, enters the brain.

SASS6 Spindle assembly abnormal protein 6 homolog. Central scaffolding component of the centrioles ensuring their ninefold symmetry. Required for centrosome biogenesis and duplication: required both for mother-centriole-dependent centriole duplication and deuterosome-dependent centriole amplification in multiciliated cells. Overexpression results in excess foci-bearing centriolar markers. Required for the recruitment of STIL to the procentriole and for STILmediated centriole amplification.

WDFY3 WD repeat and FYV domain-containing protein 3. Required for selective macroautophagy (aggrephagy). Acts as an adapter protein by linking specific proteins destined for degradation to the core autophagic machinery members, such as the ATG5-ATG12-ATG16L E3-like ligase, SQSTM1, and LC3. Along with p62/SQSTM1, involved in the formation and autophagic degradation of cytoplasmic ubiquitin-containing inclusions (p62 bodies, ALIS/ aggresome-like induced structures). Along with SQSTM1, required to recruit ubiquitinated proteins to PML bodies in the nucleus. Important for normal brain development.

ZNF335 Zinc finger protein 335. Component or associated component of some histone methyltransferase complexes that may regulate transcription through the recruitment of these complexes on gene promoters. Enhances ligand-dependent transcriptional activation by nuclear hormone receptors. Plays an important role in neural progenitor cell proliferation and self-renewal through the regulation of specific genes involved in brain development, including REST. Also controls the expressions of genes involved in somatic development and regulates, for instance, lymphoblast proliferation.

C7ORF43 Chromosome 7 open reading frame 43, with unknown function.

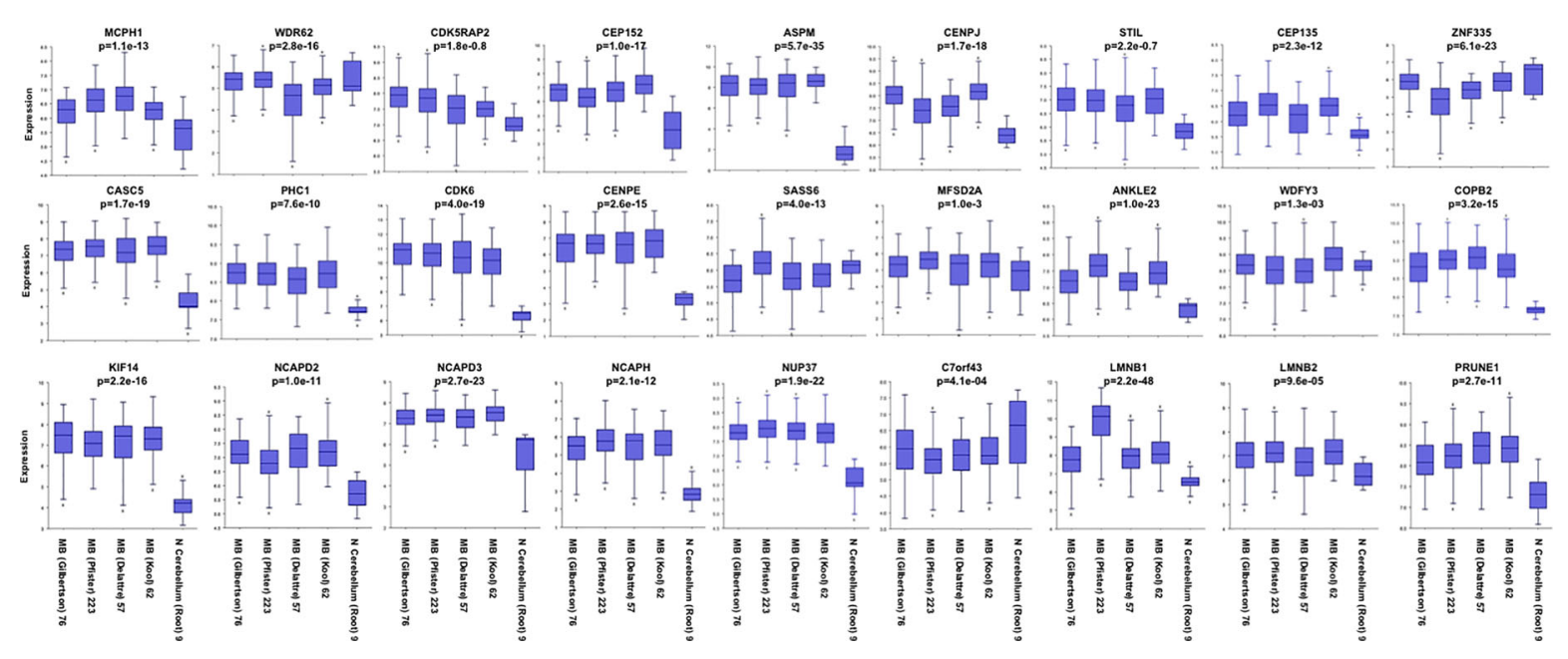

FIGURE 3 | Representative expression gene panel list of genes that have been found causative of microcephaly (MCPH). RNA log2 expression of the genes reported as causative of primary MCPH (MCPH1, WDR62, CDK5RAP2, CEP152, ASPM, CENPJ, STIL, CEP135, ZNF335, CASC5, PHC1, CDK6, CENPE, SASS6, MFSD2A, ANKLE2, WDFY3, COPB2, KIF14, NCAPD2, NCAPD3, NCAPH, NUP37, C7orf43, LMNB1, and LMNB2) and PRUNE_1 derived from transcriptome analysis of the primary cohorts of medulloblastoma (MB) in public available datasets (Pfister, Delattre, Gilbertson, and Kool) and normal cerebellum (Root) using the R2 Genomics Analysis and Visualization Platform (http://r2.amc.nl). 


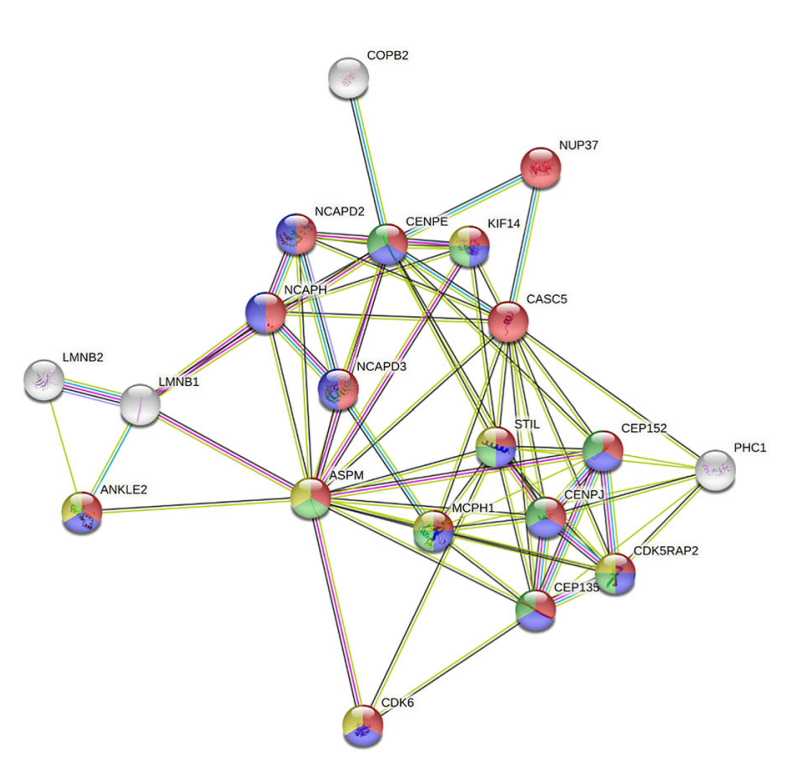

FIGURE 4 | Protein network generated via STRING (Search Tool for Retrieval of Interacting Genes/Proteins). Analyses for the STRING database used the genes reported as causative of primary microcephaly $(\mathrm{MCPH})$ and found overexpressed in medulloblastoma (MB) publicly available datasets (i.e., MCPH1, CDK5RAP2, CEP152, ASPM, CENPJ, STIL, CEP135, CASC5, PHC1, CDK6, CENPE, ANKLE2, COPB2, KIF14, NCAPD2, NCAPD3, NCAPH, NUP37, LMNB1, and LMNB2). The network is characterized by proteins involved in cell cycle (red, GO:0007049), mitotic cell cycle process (b/ue, GO:1903047), microtubule (MT)-based process (green, GO:0007017), and central nervous system (CNS) development (yellow, GO:0007417).

polymerization $(85,86)$, probably acting as a source of GTP necessary for $\alpha$ - and $\beta$-tubulins during their assembly. Altogether, these data suggest a potential role for the Prune_1 and Nm23-H1 complex during MT polymerization processes.

At this time, we can postulate that the Prune_1 protein may be implicated in both NDD and brain tumor development mostly due to its enzymatic activities and its ability to activate signaling cascades (Figure 5). Prune_1, by acting as a MAP, enhances MT polymerization, thus modulating the dynamics of MTs in the mitotic spindle during mitosis. Thus, in NMIHBA patients, mutated Prune_1 proteins were found to be responsible for the delayed MT polymerization and the decreased cell proliferation and migration processes (Figure 5, upper panel). On the other hand, in tumorigenic cells, the Prune_1 protein was found to activate canonical Wnt (via its binding to GSK-3 $\beta$ ), thus promoting the activation of $\beta$ catenin and the secretion of Wnt3a. Prune_1 was also reported to enhance the TGF- $\beta$ pathway (through interaction with Nm23-H1), increase OTX2 and N-cadherin levels, and reduce PTEN levels. Moreover, Prune_1 was reported to modulate the secretion of soluble cytokines (including IL-17F) and the vesicle protein content (e.g., vimentin). These mechanisms of action lead to the increase of the cell proliferation rate of tumorigenic cells and their metastatic spread (Figure 5, bottom panel).

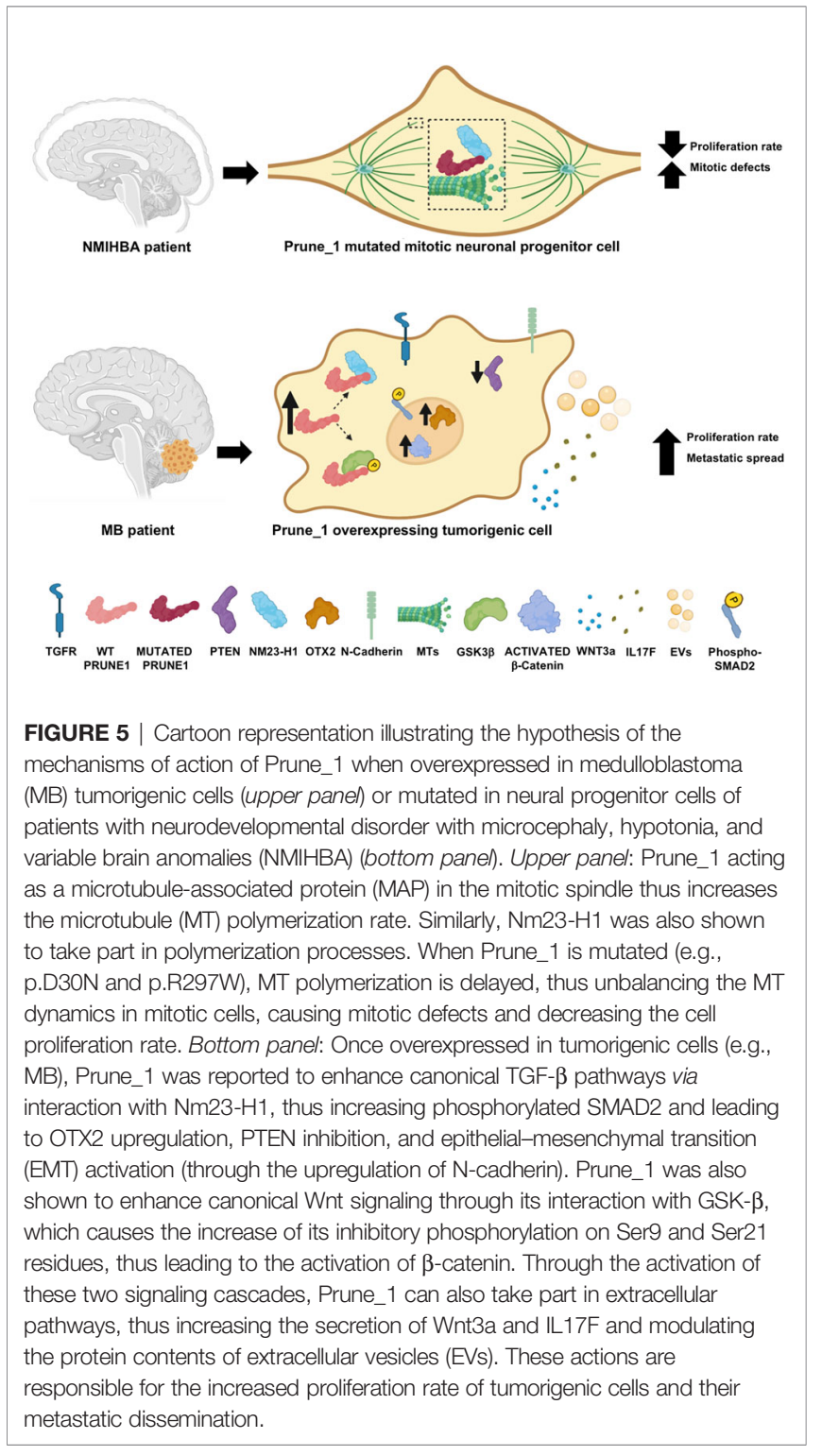

In conclusion, Prune_1 is emerging as a novel attractive target in both NDD and brain tumorigenesis. To date, several approaches (e.g., pharmacological inhibition or cell competitive permeable peptides) to impair Prune_1 protein, its enzymatic activities, or its interaction with other protein binding partners have been tested in vitro and/or in vivo in preclinical animal models (Table 2). Furthermore, the development of new small molecules able to rescue the altered enzymatic activity of mutated Prune_1 proteins could also be useful for the treatment of tumors in which the same mutations in PRUNE_1 locus were found.

Despite the promising results here presented related to the use of novel modalities of Prune_1 inhibitors, these small molecules have not been tested yet in clinical settings. We envision, at this time, that aggressive and metastatic tumors will greatly benefit from these inhibitors in new clinical programs. 


\section{AUTHOR CONTRIBUTIONS}

FB and CS performed the expression analyses and generated the protein network and the gene ontology. VF and MZ wrote the manuscript. All authors discussed the data and contributed to the final work.

\section{FUNDING}

This study was supported by PRIN Ministero dell'Università e Ricerca Italiana Project no. 2017FNZRN3 (MZ), the Italian Association for Cancer Research (AIRC; grant IG no. 22129) to MZ, Fondazione Cariplo (to VF and MZ), Fondazione Celeghin Italiana (to MZ), and Ministero dell'Università e

\section{REFERENCES}

1. Banfi S, Borsani G, Rossi E, Bernard L, Guffanti A, Rubboli F, et al. Identification and Mapping of Human cDNAs Homologous to Drosophila Mutant Genes Through EST Database Searching. Nat Genet (1996) 13 (2):167-74. doi: 10.1038/ng0696-167

2. Timmons L, Shearn A. Germline Transformation Using a Prune cDNA Rescues Prune/Killer of Prune Lethality and the Prune Eye Color Phenotype in Drosophila. Genetics (1996) 144(4):1589-600. doi: 10.1093/ genetics/144.4.1589

3. Reymond A, Volorio S, Merla G, Al-Maghtheh M, Zuffardi O, Bulfone A, et al. Evidence for Interaction Between Human PRUNE and Nm23-H1 NDPKinase. Oncogene (1999) 18(51):7244-52. doi: 10.1038/sj.onc.1203140

4. Teng DH, Engele CM, Venkatesh TR. A Product of the Prune Locus of Drosophila Is Similar to Mammalian GTPase-Activating Protein. Nature (1991) 353(6343):437-40. doi: 10.1038/353437a0

5. Carotenuto P, Marino N, Bello AM, D'Angelo A, Di Porzio U, Lombardi D, et al. PRUNE and NM23-M1 Expression in Embryonic and Adult Mouse Brain. J Bioenerg Biomembr (2006) 38(3-4):233-46. doi: 10.1007/s10863-0069044-z

6. D'Angelo A, Garzia L, Andre A, Carotenuto P, Aglio V, Guardiola O, et al. Prune cAMP Phosphodiesterase Binds Nm23-H1 and Promotes Cancer Metastasis. Cancer Cell (2004) 5(2):137-49. doi: 10.1016/S1535-6108(04) 00021-2

7. Tammenkoski M, Koivula K, Cusanelli E, Zollo M, Steegborn C, Baykov AA, et al. Human Metastasis Regulator Protein H-Prune Is a Short-Chain Exopolyphosphatase. Biochemistry (2008) 47(36):9707-13. doi: 10.1021/ bi8010847

8. Carotenuto M, De Antonellis P, Liguori L, Benvenuto G, Magliulo D, Alonzi A, et al. H-Prune Through GSK-3beta Interaction Sustains Canonical WNT/betaCatenin Signaling Enhancing Cancer Progression in NSCLC. Oncotarget (2014) 5(14):5736-49. doi: 10.18632/oncotarget.2169

9. Diana D, Smaldone G, De Antonellis P, Pirone L, Carotenuto M, Alonzi A, et al. Mapping Functional Interaction Sites of Human Prune C-Terminal Domain by NMR Spectroscopy in Human Cell Lysates. Chemistry (2013) 19 (37):12217-20. doi: 10.1002/chem.201302168

10. Zollo M, Ahmed M, Ferrucci V, Salpietro V, Asadzadeh F, Carotenuto M, et al. PRUNE Is Crucial for Normal Brain Development and Mutated in Microcephaly With Neurodevelopmental Impairment. Brain (2017) 140 (4):940-52. doi: 10.1093/brain/awx014

11. Ferrucci V, de Antonellis P, Pennino FP, Asadzadeh F, Virgilio A, Montanaro D, et al. Metastatic Group 3 Medulloblastoma Is Driven by PRUNE1 Targeting NME1-TGF-Beta-OTX2-SNAIL via PTEN Inhibition. Brain (2018) 141(5):1300-19. doi: 10.1093/brain/awy039

12. Karaca E, Harel T, Pehlivan D, Jhangiani SN, Gambin T, Coban Akdemir Z, et al. Genes That Affect Brain Structure and Function Identified by Rare Variant Analyses of Mendelian Neurologic Disease. Neuron (2015) 88(3):499513. doi: 10.1016/j.neuron.2015.09.048 della Ricerca Italiana (PRIN; grant no. 2017FNZRN3) (to MZ).

\section{ACKNOWLEDGMENTS}

We thank HAIMBIO, Seoul Korea, in the name of Dr. HongYeoul Kim, for sharing ideas and compounds to be used as inhibitor of Prune_1 to reduce the activated mitochondrial cancer metabolism and for studies related to the use of polyPs. We further thank Prof. Werner Muller for helpful discussion on PolyPs and Sars-Cov-2 infection and replication in the context of Prune_1 overexpression. We also thank Associazione S.P.R.IN.T. Onlus for supporting research on Prune_1 and other tubulinopathy genes involving microtubule dynamics.

13. Costain G, Shugar A, Krishnan P, Mahmutoglu S, Laughlin S, Kannu P. Homozygous Mutation in PRUNE1 in an Oji-Cree Male With a Complex Neurological Phenotype. Am J Med Genet A (2017) 173(3):740-3. doi: 10.1002/ajmg.a.38066

14. Iacomino M, Fiorillo C, Torella A, Severino M, Broda P, Romano C, et al. Spinal Motor Neuron Involvement in a Patient With Homozygous PRUNE Mutation. Eur J Paediatr Neurol (2018) 22(3):541-3. doi: 10.1016/j.ejpn.2017.12.005

15. Alfadhel M, Nashabat M, Hundallah K, Al Hashem A, Alrumayyan A, Tabarki B. PRUNE Syndrome Is a New Neurodevelopmental Disorder: Report and Review. Child Neurol Open (2018) 5:2329048X17752237. doi: 10.1177/ 2329048X17752237

16. Alhaddad B, Schossig A, Haack TB, Kovacs-Nagy R, Braunisch MC, Makowski C, et al. PRUNE1 Deficiency: Expanding the Clinical and Genetic Spectrum. Neuropediatrics (2018) 49(5):330-8. doi: 10.1055/s-00381661396

17. Imagawa E, Yamamoto Y, Mitsuhashi S, Isidor B, Fukuyama T, Kato M, et al. PRUNE1-Related Disorder: Expanding the Clinical Spectrum. Clin Genet (2018) 94(3-4):362-7. doi: 10.1111/cge.13385

18. Okur D, Daimaguler HS, Danyeli AE, Tekgul H, Wang H, Wunderlich G, et al. Bi-Allelic Mutations in PRUNE Lead to Neurodegeneration With Spinal Motor Neuron Involvement and Hyperckaemia. Turk J Pediatr (2019) 61 (6):931-6. doi: 10.24953/turkjped.2019.06.015

19. Papuc SM, Abela L, Steindl K, Begemann A, Simmons TL, Schmitt B, et al. The Role of Recessive Inheritance in Early-Onset Epileptic Encephalopathies: A Combined Whole-Exome Sequencing and Copy Number Study. Eur J Hum Genet (2019) 27(3):408-21. doi: 10.1038/s41431-018-0299-8

20. Fujii H, Sato N, Takanashi JI, Kimura Y, Morimoto E, Shigemoto Y, et al. Altered MR Imaging Findings in a Japanese Female Child With PRUNE1-Related Disorder. Brain Dev (2020) 42(3):302-6. doi: 10.1016/j.braindev.2019.12.001

21. Essayan DM. Cyclic Nucleotide Phosphodiesterases. J Allergy Clin Immunol (2001) 108(5):671-80. doi: 10.1067/mai.2001.119555

22. Spano D, Marshall JC, Marino N, De Martino D, Romano A, Scoppettuolo $\mathrm{MN}$, et al. Dipyridamole Prevents Triple-Negative Breast-Cancer Progression. Clin Exp Metastasis (2013) 30(1):47-68. doi: 10.1007/s10585-012-9506-0

23. Virgilio A, Spano D, Esposito V, Di Dato V, Citarella G, Marino N, et al. Novel Pyrimidopyrimidine Derivatives for Inhibition of Cellular Proliferation and Motility Induced by H-Prune in Breast Cancer. Eur J Med Chem (2012) 57:4150. doi: 10.1016/j.ejmech.2012.08.020

24. Simbulan-Rosenthal CM, Gaur A, Sanabria VA, Dussan LJ, Saxena R, Schmidt J, et al. Inorganic Polyphosphates Are Important for Cell Survival and Motility of Human Skin Keratinocytes. Exp Dermatol (2015) 24(8):636-9. doi: 10.1111/exd.12729

25. Bondy-Chorney E, Abramchuk I, Nasser R, Holinier C, Denoncourt A, Baijal $\mathrm{K}$, et al. A Broad Response to Intracellular Long-Chain Polyphosphate in Human Cells. Cell Rep (2020) 33(4):108318. doi: 10.1016/j.celrep.2020.108318

26. Morrissey JH. Polyphosphate: A Link Between Platelets, Coagulation and Inflammation. Int J Hematol (2012) 95(4):346-52. doi: 10.1007/s12185-0121054-5 
27. Muller WEG, Schroder HC, Wang X. Inorganic Polyphosphates As Storage for and Generator of Metabolic Energy in the Extracellular Matrix. Chem Rev (2019) 119(24):12337-74. doi: 10.1021/acs.chemrev.9b00460

28. Lorenz B, Leuck J, Kohl D, Muller WE, Schroder HC. Anti-HIV-1 Activity of Inorganic Polyphosphates. J Acquir Immune Defic Syndr Hum Retrovirol (1997) 14(2):110-8. doi: 10.1097/00042560-199702010-00003

29. Ferrucci V, Kong DY, Asadzadeh F, Marrone L, Boccia A, Siciliano R, et al. Long-Chain Polyphosphates Impair SARS-CoV-2 Infection and Replication. Sci Signal (2021) 14(690). doi: 10.1126/scisignal.abe5040

30. Zollo M, Ferrucci V, Izzo B, Quarantelli F, Domenico CD, Comegna M, et al. SARS-CoV-2 Subgenomic N (sgN) Transcripts in Oro-Nasopharyngeal Swabs Correlate with the Highest Viral Load, as Evaluated by Five Different Molecular Methods. Diagnostics (Basel) (2021) 11(2):288. doi: 10.3390/ diagnostics 11020288

31. Angelova PR, Agrawalla BK, Elustondo PA, Gordon J, Shiba T, Abramov AY, et al. In Situ Investigation of Mammalian Inorganic Polyphosphate Localization Using Novel Selective Fluorescent Probes JC-D7 and JC-D8. ACS Chem Biol (2014) 9(9):2101-10. doi: 10.1021/cb5000696

32. Holmstrom KM, Marina N, Baev AY, Wood NW, Gourine AV, Abramov AY. Signalling Properties of Inorganic Polyphosphate in the Mammalian Brain. Nat Commun (2013) 4:1362. doi: 10.1038/ncomms2364

33. Dinarvand P, Hassanian SM, Qureshi SH, Manithody C, Eissenberg JC, Yang L, et al. Polyphosphate Amplifies Proinflammatory Responses of Nuclear Proteins Through Interaction With Receptor for Advanced Glycation End Products and P2Y1 Purinergic Receptor. Blood (2014) 123(6):935-45. doi: 10.1182/blood-2013-09-529602

34. Abramov AY, Fraley C, Diao CT, Winkfein R, Colicos MA, Duchen MR, et al. Targeted Polyphosphatase Expression Alters Mitochondrial Metabolism and Inhibits Calcium-Dependent Cell Death. Proc Natl Acad Sci USA (2007) 104 (46):18091-6. doi: 10.1073/pnas.0708959104

35. Pavlov E, Aschar-Sobbi R, Campanella M, Turner RJ, Gomez-Garcia MR, Abramov AY. Inorganic Polyphosphate and Energy Metabolism in Mammalian Cells. J Biol Chem (2010) 285(13):9420-8. doi: 10.1074/ jbc.M109.013011

36. Nakamura A, Kawano N, Motomura K, Kuroda A, Sekiguchi K, Miyado M, et al. Degradation of Phosphate Polymer polyP Enhances Lactic Fermentation in Mice. Genes Cells (2018) 23(10):904-14. doi: 10.1111/gtc.12639

37. Carotenuto M, Pedone E, Diana D, de Antonellis P, Dzeroski S, Marino N, et al. Neuroblastoma Tumorigenesis Is Regulated Through the Nm23-H1/hPrune C-Terminal Interaction. Sci Rep (2013) 3:1351. doi: 10.1038/srep01351

38. Middelhaufe S, Garzia L, Ohndorf UM, Kachholz B, Zollo M, Steegborn C. Domain Mapping on the Human Metastasis Regulator Protein H-Prune Reveals a C-Terminal Dimerization Domain. Biochem J (2007) 407(2):199205. doi: $10.1042 / B J 20070408$

39. Marino N, Zollo M. Understanding H-Prune Biology in the Fight Against Cancer. Clin Exp Metastasis (2007) 24(8):637-45. doi: 10.1007/s10585-007-9109-3

40. Kobayashi T, Hino S, Oue N, Asahara T, Zollo M, Yasui W, et al. Glycogen Synthase Kinase 3 and H-Prune Regulate Cell Migration by Modulating Focal Adhesions. Mol Cell Biol (2006) 26(3):898-911. doi: 10.1128/MCB.26.3.898911.2006

41. Garzia L, D'Angelo A, Amoresano A, Knauer SK, Cirulli C, Campanella C, et al. Phosphorylation of Nm23-H1 by CKI Induces Its Complex Formation With H-Prune and Promotes Cell Motility. Oncogene (2008) 27(13):1853-64. doi: $10.1038 /$ sj.onc. 1210822

42. Garzia L, Roma C, Tata N, Pagnozzi D, Pucci P, Zollo M. H-Prune-Nm23-H1 Protein Complex and Correlation to Pathways in Cancer Metastasis. $J$ Bioenerg Biomembr (2006) 38(3-4):205-13. doi: 10.1007/s10863-006-9036-Z

43. Freeman J, Smith D, Latinkic B, Ewan K, Samuel L, Zollo M, et al. A Functional Connectome: Regulation of Wnt/TCF-Dependent Transcription by Pairs of Pathway Activators. Mol Cancer (2015) 14:206. doi: 10.1186/ s12943-015-0475-1

44. Ferrucci V, Pennino FP, Siciliano R, Asadzadeh F, Zollo M. A Competitive Cell-Permeable Peptide Impairs Nme-1 (NDPK-A) and Prune-1 Interaction: Therapeutic Applications in Cancer. Lab Invest (2018) 98(5):571-81. doi: 10.1038/s41374-017-0011-6

45. Zollo M, Andre A, Cossu A, Sini MC, D'Angelo A, Marino N, et al. Overexpression of H-Prune in Breast Cancer Is Correlated With Advanced Disease Status. Clin Cancer Res (2005) 11(1):199-205.
46. Galasso A, Zollo M. The Nm23-H1-H-Prune Complex in Cellular Physiology: A 'Tip of the Iceberg' Protein Network Perspective. Mol Cell Biochem (2009) 329(1-2):149-59. doi: 10.1007/s11010-009-0115-4

47. Siskos N, Stylianopoulou E, Skavdis G, Grigoriou ME. Molecular Genetics of Microcephaly Primary Hereditary: An Overview. Brain Sci (2021) 11(5). doi: 10.3390/brainsci11050581

48. Bahi-Buisson N, Poirier K, Fourniol F, Saillour Y, Valence S, Lebrun N, et al. The Wide Spectrum of Tubulinopathies: What Are the Key Features for the Diagnosis? Brain (2014) 137(Pt 6):1676-700. doi: 10.1093/brain/awu082

49. Bilitou A, De Marco N, Bello AM, Garzia L, Carotenuto P, Kim M, et al. Spatial and Temporal Expressions of Prune Reveal a Role in Muller Gliogenesis During Xenopus Retinal Development. Gene (2012) 509(1):93103. doi: 10.1016/j.gene.2012.08.001

50. Koko M, Yahia A, Elsayed LE, Hamed AA, Mohammed IN, Elseed MA, et al. An Identical-by-Descent Novel Splice-Donor Variant in PRUNE1 Causes a Neurodevelopmental Syndrome With Prominent Dystonia in Two Consanguineous Sudanese Families. Ann Hum Genet (2021) 85(5):186-95. doi: 10.1111/ahg. 12437

51. Hartley JN, Simard LR, Ly V, Del Bigio MR, Frosk P. A Homozygous Canonical Splice Acceptor Site Mutation in PRUNE1 Is Responsible for a Rare Childhood Neurodegenerative Disease in Manitoba Cree Families. Am J Med Genet A (2019) 179(2):206-18. doi: 10.1002/ajmg.a.60690

52. Karakaya M, Yilmaz S, Storbeck M, Hoelker I, Heller R, Serdaroglu G, et al. PRUNE1: A Disease-Causing Gene for Secondary Microcephaly. Brain (2017) 140(10):e61. doi: 10.1093/brain/awx197

53. Mehdi Agha Gholizadeh MM-S, Fardanesh F, Garshasbi M. Neurodevelopmental Disorder With Microcephaly, Hypotonia, And Variable Brain Anomalies In A Consanguineous Iranian Family Is Associated With A Novel Homozygous Start Loss Variant In The PRUNE1 Gene. In Review. BMC Med Genomics (2021). doi: 10.21203/rs.3.rs-789457/v1

54. Nistala H, Dronzek J, Gonzaga-Jauregui C, Chim SM, Rajamani S, Nuwayhid S, et al. NMIHBA Results From Hypomorphic PRUNE1 Variants That Lack Short-Chain Exopolyphosphatase Activity. Hum Mol Genet (2021) 29 (21):3516-31. doi: 10.1093/hmg/ddaa237

55. Oue N, Yoshida K, Noguchi T, Sentani K, Kikuchi A, Yasui W. Increased Expression of H-Prune Is Associated With Tumor Progression and Poor Survival in Gastric Cancer. Cancer Sci (2007) 98(8):1198-205. doi: 10.1111/ j.1349-7006.2007.00515.x

56. Noguchi T, Oue N, Wada S, Sentani K, Sakamoto N, Kikuchi A, et al. H-Prune Is an Independent Prognostic Marker for Survival in Esophageal Squamous Cell Carcinoma. Ann Surg Oncol (2009) 16(5):1390-6. doi: 10.1245/s10434-007-9585-3

57. Nambu J, Kobayashi T, Hashimoto M, Tashiro H, Sugino K, Shimamoto F, et al. H-Prune Affects Anaplastic Thyroid Cancer Invasion and Metastasis. Oncol Rep (2016) 35(6):3445-52. doi: 10.3892/or.2016.4759

58. Hashimoto M, Kobayashi T, Tashiro H, Arihiro K, Kikuchi A, Ohdan H. HPrune Is Associated With Poor Prognosis and Epithelial-Mesenchymal Transition in Patients With Colorectal Liver Metastases. Int J Cancer (2016) 139(4):812-23. doi: 10.1002/ijc.30118

59. Ferrucci V, Asadzadeh F, Collina F, Siciliano R, Boccia A, Marrone L, et al. Prune-1 Drives Polarization of Tumor-Associated Macrophages (TAMs) Within the Lung Metastatic Niche in Triple-Negative Breast Cancer. iScience (2021) 24(1):101938. doi: 10.1016/j.isci.2020.101938

60. Cavalli FMG, Remke M, Rampasek L, Peacock J, Shih DJH, Luu B, et al. Intertumoral Heterogeneity Within Medulloblastoma Subgroups. Cancer Cell (2017) 31(6):737-754 e6. doi: 10.1016/j.ccell.2017.05.005

61. Silva GO, He X, Parker JS, Gatza ML, Carey LA, Hou JP, et al. Cross-Species DNA Copy Number Analyses Identifies Multiple 1q21-Q23 Subtype-Specific Driver Genes for Breast Cancer. Breast Cancer Res Treat (2015) 152(2):34756. doi: 10.1007/s10549-015-3476-2

62. Goh JY, Feng M, Wang W, Oguz G, Yatim S, Lee PL, et al. Chromosome 1q21.3 Amplification Is a Trackable Biomarker and Actionable Target for Breast Cancer Recurrence. Nat Med (2017) 23(11):1319-30. doi: 10.1038/ nm. 4405

63. Northcott PA, Korshunov A, Witt H, Hielscher T, Eberhart CG, Mack S, et al. Medulloblastoma Comprises Four Distinct Molecular Variants. J Clin Oncol (2011) 29(11):1408-14. doi: 10.1200/JCO.2009.27.4324

64. Kool M, Korshunov A, Remke M, Jones DT, Schlanstein M, Northcott PA, et al. Molecular Subgroups of Medulloblastoma: An International Meta- 
Analysis of Transcriptome, Genetic Aberrations, and Clinical Data of WNT, SHH, Group 3, and Group 4 Medulloblastomas. Acta Neuropathol (2012) 123 (4):473-84. doi: 10.1007/s00401-012-0958-8

65. Sharma T, Schwalbe EC, Williamson D, Sill M, Hovestadt V, Mynarek M, et al. Second-Generation Molecular Subgrouping of Medulloblastoma: An International Meta-Analysis of Group 3 and Group 4 Subtypes. Acta Neuropathol (2019) 138(2):309-26. doi: 10.1007/s00401-019-02020-0

66. Hovestadt V, Smith KS, Bihannic L, Filbin MG, Shaw ML, Baumgartner A, et al. Resolving Medulloblastoma Cellular Architecture by Single-Cell Genomics. Nature (2019) 572(7767):74-9. doi: 10.1038/s41586-019-1434-6

67. Robinson GW, Orr BA, Wu G, Gururangan S, Lin T, Qaddoumi I, et al. Vismodegib Exerts Targeted Efficacy Against Recurrent Sonic HedgehogSubgroup Medulloblastoma: Results From Phase II Pediatric Brain Tumor Consortium Studies PBTC-025B and PBTC-032. J Clin Oncol (2015) 33 (24):2646-54. doi: 10.1200/JCO.2014.60.1591

68. Goschzik T, Schwalbe EC, Hicks D, Smith A, Zur Muehlen A, FigarellaBranger D, et al. Prognostic Effect of Whole Chromosomal Aberration Signatures in Standard-Risk, Non-WNT/Non-SHH Medulloblastoma: A Retrospective, Molecular Analysis of the HIT-SIOP PNET 4 Trial. Lancet Oncol (2018) 19(12):1602-16. doi: 10.1016/S1470-2045(18)30532-1

69. Gajjar AJ, Robinson GW. Medulloblastoma-Translating Discoveries From the Bench to the Bedside. Nat Rev Clin Oncol (2014) 11(12):714-22. doi: 10.1038/ nrclinonc.2014.181

70. Hill RM, Kuijper S, Lindsey JC, Petrie K, Schwalbe EC, Barker K, et al. Combined MYC and P53 Defects Emerge at Medulloblastoma Relapse and Define Rapidly Progressive, Therapeutically Targetable Disease. Cancer Cell (2015) 27(1):72-84. doi: 10.1016/j.ccell.2014.11.002

71. Asadzadeh F, Ferrucci V, DE Antonellis P, Zollo M. In Vivo Bioluminescence Imaging Using Orthotopic Xenografts Towards Patient's Derived-Xenograft Medulloblastoma Models. Q J Nucl Med Mol Imaging (2017) 61(1):95-101. doi: 10.23736/S1824-4785.16.02959-9

72. Liao H, Liao M, Xu L, Yan X, Ren B, Zhu Z, et al. Integrative Analysis of HPrune as a Potential Therapeutic Target for Hepatocellular Carcinoma. EBioMedicine (2019) 41:310-9. doi: 10.1016/j.ebiom.2019.01.001

73. Carotenuto M, de Antonellis P, Chiarolla CM, Attanasio C, Damiani V, Boffa I, et al. A Therapeutic Approach to Treat Prostate Cancer by Targeting Nm23H1/h-Prune Interaction. Naunyn Schmiedebergs Arch Pharmacol (2015) 388 (2):257-69. doi: 10.1007/s00210-014-1035-8

74. Lee JJ, Kim HS, Lee JS, Park J, Shin SC, Song S, et al. Small Molecule Activator of Nm23/NDPK as an Inhibitor of Metastasis. Sci Rep (2018) 8(1):10909. doi: 10.1038/s41598-018-29101-6

75. Han KY, Hong BS, Yoon YJ, Yoon CM, Kim YK, Kwon YG, et al. Polyphosphate Blocks Tumour Metastasis via Anti-Angiogenic Activity. Biochem J (2007) 406(1):49-55. doi: 10.1042/BJ20061542

76. Tsutsumi K, Matsuya Y, Sugahara T, Tamura M, Sawada S, Fukura S, et al. Inorganic Polyphosphate Enhances Radio-Sensitivity in a Human Non-Small Cell Lung Cancer Cell Line, H1299. Tumour Biol (2017) 39 (6):1010428317705033. doi: 10.1177/1010428317705033

77. Budday S, Steinmann P, Kuhl E. Physical Biology of Human Brain Development. Front Cell Neurosci (2015) 9:257. doi: 10.3389/fncel.2015.00257
78. Lang PY, Gershon TR. A New Way to Treat Brain Tumors: Targeting Proteins Coded by Microcephaly Genes?: Brain Tumors and Microcephaly Arise From Opposing Derangements Regulating Progenitor Growth. Drivers of Microcephaly Could be Attractive Brain Tumor Targets . Bioessays (2018) 40(5):e1700243. doi: 10.1002/bies.201700243

79. Vladoiu MC, El-Hamamy I, Donovan LK, Farooq H, Holgado BL, Sundaravadanam Y, et al. Childhood Cerebellar Tumours Mirror Conserved Fetal Transcriptional Programs. Nature (2019) 572(7767):67-73. doi: 10.1038/s41586-019-1158-7

80. Dirks PB. Brain Tumor Stem Cells: Bringing Order to the Chaos of Brain Cancer. J Clin Oncol (2008) 26(17):2916-24. doi: 10.1200/JCO.2008.17.6792

81. Shain AH, Pollack JR. The Spectrum of SWI/SNF Mutations, Ubiquitous in Human Cancers. PloS One (2013) 8(1):e55119. doi: 10.1371/journal.pone.0055119

82. Shain AH, Giacomini CP, Matsukuma K, Karikari CA, Bashyam MD, Hidalgo $\mathrm{M}$, et al. Convergent Structural Alterations Define SWItch/Sucrose NonFermentable (SWI/SNF) Chromatin Remodeler as a Central Tumor Suppressive Complex in Pancreatic Cancer. Proc Natl Acad Sci USA (2012) 109(5):E252-9. doi: 10.1073/pnas.1114817109

83. Jones DT, Jager N, Kool M, Zichner T, Hutter B, Sultan M, et al. Dissecting the Genomic Complexity Underlying Medulloblastoma. Nature (2012) 488 (7409):100-5. doi: 10.1038/nature11284

84. Roymans D, Vissenberg K, De Jonghe C, Willems R, Engler G, Kimura N, et al. Identification of the Tumor Metastasis Suppressor Nm23-H1/Nm23-R1 as a Constituent of the Centrosome. Exp Cell Res (2001) 262(2):145-53. doi: 10.1006/excr.2000.5087

85. Lombardi D, Sacchi A, D'Agostino G, Tibursi G. The Association of the Nm23-M1 Protein and Beta-Tubulin Correlates With Cell Differentiation. Exp Cell Res (1995) 217(2):267-71. doi: 10.1006/excr.1995.1086

86. Pinon VP, Millot G, Munier A, Vassy J, Linares-Cruz G, Capeau J, et al. Cytoskeletal Association of the A and B Nucleoside Diphosphate Kinases of Interphasic But Not Mitotic Human Carcinoma Cell Lines: Specific Nuclear Localization of the B Subunit. Exp Cell Res (1999) 246(2):355-67. doi: 10.1006/ excr.1998.4318

Conflict of Interest: The authors declare that the research was conducted in the absence of any commercial or financial relationships that could be construed as a potential conflict of interest.

Publisher's Note: All claims expressed in this article are solely those of the authors and do not necessarily represent those of their affiliated organizations, or those of the publisher, the editors and the reviewers. Any product that may be evaluated in this article, or claim that may be made by its manufacturer, is not guaranteed or endorsed by the publisher.

Copyright $\odot 2021$ Bibbò, Sorice, Ferrucci and Zollo. This is an open-access article distributed under the terms of the Creative Commons Attribution License (CC BY). The use, distribution or reproduction in other forums is permitted, provided the original author(s) and the copyright owner(s) are credited and that the original publication in this journal is cited, in accordance with accepted academic practice. No use, distribution or reproduction is permitted which does not comply with these terms. 